\title{
Intra-Articular Injection of Autologous Adipose-Derived Stromal Vascular Fractions for the Cartilage Repair of Grade 2 and 3 Knee Osteoarthritis: A Confirmatory Clinical Trial
}

\section{Yin Zhang}

Zhejiang Provincial People's Hospital https://orcid.org/0000-0002-9742-5421

\section{Qing Bi}

Zhejiang Provincial People's Hospital

Taihen Yu

Zhejiang Provincial People's Hospital

\section{Zheping Hong}

Zhejiang Provincial People's Hospital

\section{Yu Tong}

Zhejiang Provincial People's Hospital

Junchao Luo

Wenzhou Medical University

Ye Yuan

Zhejiang Provincial People's Hospital

\section{Wei Zhang}

Zhejiang Provincial People's Hospital

\section{Senbo Zhu}

Wenzhou Medical University

Lichen Ji

Wenzhou Medical University

\section{Chen Zhao}

Zhejiang Provincial People's Hospital

\section{Shuijun Zhang}

Zhejiang Provincial People's Hospital

Qiong Zhang ( $\nabla$ zqzjsrmyy@163.com )

Zhejiang Provincial People's Hospital

\section{Research}

Keywords: Adipose-derived stromal vascular fractions, Cartilage regeneration, 3D-FS-SPGR sequence, Intra-articular injection, Knee osteoarthritis

Posted Date: February 15th, 2021

DOI: https://doi.org/10.21203/rs.3.rs-193150/v1

License: (9) (i) This work is licensed under a Creative Commons Attribution 4.0 International License. Read Full License 


\section{Abstract}

\section{Background}

Cartilage defect remains one of the most important reasons for the rapid development of knee osteoarthritis (OA). Numerous reports have confirmed the safety and clinical efficacy of autologous adipose-derived stromal vascular fractions (SVF), which has recently been used clinically to treat patients with knee OA. However, there is still no consensus as to whether SVF can promote cartilage regeneration. Herein, we purposed to evaluate the effectiveness of SVF in cartilage regeneration by developing cartilage model based on the 3D-FS-SPGR sequence.

Methods

Patients with Kellgren-Lawrence grade 2-3 knee OA were recruited in our research. Then, we monitored patients and subsequently scored symptoms using WOMAC, VAS, and range of motion (ROM) before treatment and at 1, 3, 6, and 12 months post-treatment. The WORMS and MOCART were recorded by magnetic resonance imaging. The cartilage model of the patient was established using the 3D-FS-SPGR sequence, while the relevant parameters of the model were counted at baseline, 6 , and 12 months.

Results

We enrolled 47 patients (53 knees) with knee OA in this study, of which 29 knees were classified as grade 2, while 24 were assigned grade 3. No treatment-related adverse event was observed in our study. Notably, WOMAC, VAS, and ROM showed a significant improvement at 12 months. We further found that the thickness, volume, and surface of the cartilage defect decreased, while the volume of healthy cartilage increased in all regions, particularly in the medial femoral and tibia condyle. Moreover, the scores of WORMS and MOCART revealed a substantial improvement of cartilage repair at 12 months.

\section{Conclusions}

Taken together, this study shows that intra-articular injection of SVF into the knee markedly improved the clinical symptoms of patients without the occurrence of adverse events, thereby repairing the damaged articular cartilage through cartilage regeneration.

Trial registration

Retrospectively registered. Chinses Clinical Trial Registry with identifier ChiCTR2100042930. Registered 28 January 2021.

\section{Background}

Osteoarthritis (OA) is a common chronic disease of the joints, which is characterized by osteophyte formation, changes to the subchondral bone, degeneration of ligaments and menisci, pain, stiffness, and loss of joint function [1, 2]. Several studies have established that knee $\mathrm{OA}$ is a highly prevalent form of arthritis that contributes to arthralgia and disability, especially in elderly people [3].

To date, more than 50 therapies of pharmacological, non-pharmacological, and surgical have been documented by scholars. However, most patients cannot inevitably avoid taking the road of total knee arthroplasty (TKA) in the end $[4,5]$. This is because these treatments can only relieve the clinical symptoms of patients, but cannot stop the degeneration of articular cartilage, leave alone regenerate them. In this respect, it is therefore of great significance to find a new and effective therapy for alleviating the clinical symptoms of osteoarthritis and preventing the degeneration of articular cartilage.

Since the discovery of the multipotent stem cell population in adipose tissue by Zuk et al, cell-based regenerative therapy has gradually become a possible method for cartilage regeneration [6]. Recent related studies have also confirmed that mesenchymal stem cells (MSCs) and adipose-derived stem cells (ADSCs) possess the potential to differentiate into 
chondrocytes. The isolation of both MSCs and ADSCs requires to take several weeks in a specialized laboratory for cell expansion $[7,8]$. However, the expansion and culture of cells significantly increase the economic burden of treatment to patients. Some scholars have proposed a more effective method to collect and manage ADSCs using stromal vascular fraction (SVF) [9]. Furthermore, adipose-derived SVF comprises numerous regenerative cells, such as ADSCs, blood cells, pericytes, fibroblasts, macrophages, smooth muscle cells, endothelial cells, and their precursors. It also exhibits the benefits of easy isolation and use without culturing or differentiation [10-13]. Studies have shown molecules of interleukin-10, interleukin-1, interleukin-1 receptor antagonist, prostaglandin E2, and transforming growth factor- $\beta$, released by SVF can exert anti-inflammatory effects on chondrocytes and synovial cells, consequently responding to the local environment of OA [14, 15]. Multiple recent reports have proved that the use of intra-articular SVF injections can effectively relieve the clinical symptoms of patients [16-19]. Nonetheless, despite these intriguing results, it remains unclear whether the SVF injections can promote regeneration of the articular cartilage, requiring further exploration. Inconsistent findings have been reported regarding this topic, whereby some studies reported evidence of cartilage tissue regeneration, while others claimed that no change is observed [20-23].

At present, there are no published clinical trials on quantitative analysis at three-dimensional (3D) level about whether SVF can promote cartilage regeneration. Most radiological evaluations of SVF remain at two-dimensional (2D) level, thus they cannot accurately examine the efficacy of cartilage repair, while arthroscopy will cause unnecessary damage to patients. Therefore, how to judge the repair of the articular cartilage at the 3D level is worth exploring. We thus designed a clinical trial about autologous adipose-derived SVF in the treatment of patients with knee OA Kellgren Lawrence (K-L) grade 2 and 3 [24]. This study sought to establish 3D cartilage models by use of a special sequence of magnetic resonance images (MRI) to quantitatively examine the effect of intra-articular SVF injections on cartilage regeneration. We divided the knee cartilage in detail and observed the changes of cartilage repair in each region. Our secondary aim was to observed different responses to SVF injection in patients with K-L grade 2 and 3. In this regard, we hypothesize that patients with intra-articular SVF injection could show significant improvement in symptoms, and the cartilage regeneration could be confirmed using quantitative analysis at the 3D level.

\section{Methods}

\section{Inclusion and exclusion criteria}

All experimental protocols used in this study were approved by the Ethics Committees of Zhejiang Provincial People's Hospital. Patients enrolled in this study provided signed written informed consent. Eligible patients included were aged between 18 and 75 years, with OA K-L grades 2 and 3, exhibiting substantial pain and loss of function, failure of conservative therapy, and had an initial pain evaluated at four or greater on a ten-point VAS in the knee joint. On the other hand, exclusion

criteria comprised of secondary arthritis (for example, secondary knee osteoarthritis, rheumatoid arthritis, gouty arthritis, and previous articular fractures), contraindicating MRI examination, a history of liposarcoma and other cancers, intra-articular injection of hyaluronic acid or other drugs in the preceding 2 months, end-stage (grade 4) OA, serological positive of Human Immunodeficiency Virus, abdominal hernia, and coagulopathy.

\section{Study design}

Procedures related to screening, assessment, fat collection, and SVF injection of patients were performed in private physician examination rooms. Three senior radiologists were responsible for the radiological evaluation and came to a consensus conclusion. The cartilage model was established by professional engineers with the help of a special MRI sequence. During scheduled clinical visits, relevant questionnaires were collected.

To evaluate the grade of OA, an initial X-ray image was used following the K-L criteria, and subsequently, patients belonging to grades 2 and 3 were selected. Afterward, patients who underwent MRI included conventional and three-dimensional fatsuppressed spoiled gradient recalled echo (3D-FS-SPGR) sequences. We used the conventional sequence to record the 
whole-organ magnetic resonance imaging score (WORMS) and magnetic resonance observation of cartilage repair tissue (MOCART), while the 3D-FS-SPGR sequence was employed to build a 3D cartilage model and measure the related parameters. Using visual analog scale (VAS) and Western Ontario and McMaster Universities Osteoarthritis Index (WOMAC) questionnaire, pain and functional limitation were evaluated. We also examined the range of motion (ROM) during the follow-up period.

\section{Clinical evaluation}

Here, the VAS and WOMAC questionnaires were used for the evaluation of pain and functional limitation. In particular, we evaluated the WOMAC score in terms of pain, stiffness, and physical function with a total score ranging from 0 (best health) to 96 (worst health). The maximum score of each subscale was 20, 8, and 68, respectively. The total score of VAS ranged from 0 (best) to 10 (worst). Additionally, we recorded the ROM of the knee joint during the follow-up. Assessments were performed at baseline (before the first injection), 1 month after injection, and during follow-up visits after 3,6 , and 12 months. Finally, we assessed the safety of SVF by analyzing the incidence rate of adverse events (AE) and serious adverse events (SAE).

\section{Radiological assessment}

For this clinical study, we performed MRI at baseline, 6, and 12 months. In order to minimize the influence of knee joint loading on the results of MRI, patients were required to rest for 30 minutes before examination. The conventional MRI sequences included T1W1 images in the sagittal plane and PDWI-FS images in the sagittal, coronal, and transverse planes. We employed the MOCART score to examine the cartilage repair, while the WORMS was used for the assessment of the knee $[25,26]$.

\section{Establishment of 3D cartilage model}

The MRI scanning was performed on a clinical 3.0T system (GE Healthcare, Waukesha, WI, USA). Using the 3D-FS-SPGR sequence, each patient was examined before SVF injection. Acquisition parameters for the 3D-FS-SPGR sequence were as follows: TE: $3 \mathrm{~ms}$; TR: $14.6 \mathrm{~ms}$; acquisition matrix; 512 x 512; the number of excitations: 2; field-of-view: $15 \mathrm{~cm}$; slice thickness: $1.0 \mathrm{~mm}$; interslice gaps: $0 \mathrm{~mm}$; and receiver $\mathrm{BW} \pm 41.7 \mathrm{kHz}$ [27].

To build the 3D cartilage model, the original data of the 3D-FS-SPGR sequence was converted to Digital Imaging and Communications in Medicine (DICOM) format and transferred into the Mimics 20.0 software (Materialise, Leuven, Belgium). First, all layers of cartilage defects were detected using 3D-FS-SPGR and conventional sequences. An appropriate segmentation threshold (1849-3445 GV) was set for retaining the healthy cartilage of the knee joint, saving the results as the green mask. Following this, the cartilage defect was segmented by another mask, then saving it as a red mask. The healthy cartilage and cartilage defects of the knee joint segmented by use of different masks are given in Fig. 1. After the layer-bylayer hierarchical image processing, the cartilage model was characterized into six regions, namely, medial femoral condyle (MF), lateral femoral condyle (LF), femoral inter-condylar (T), medial tibia condyle (MT), lateral tibia condyle (LT), and patella (P) [28]. Different color masks represented different areas, while cartilage defects were represented by red masks (Fig. 2a). Then, the cartilage tissue for each layer was preserved, the contours of knee cartilage were calculated, and the cartilage model of each region was established (Fig. 2b). The volume of healthy cartilage, as well as the volume, surface, and thickness of cartilage defects, were measured by the same professional surveyor (Fig. 2c). After one week, the cartilage model was re-established, and the above-mentioned data were measured and averaged.

\section{Fat tissue harvest}

For this experiment, patients were not allowed to take aspirin, corticosteroids, and non-steroidal anti-inflammatory drugs within one week before liposuction. Also, they all fasted for liquids and solids at least six hours before the operation. After confirmation of the eligibility criteria, the operator, anesthesiologist, and circulating nurse performed general anesthesia in 
patients in the supine position. The operation was performed by the same skilled plastic surgeon, who was blinded to patient information. After adequate disinfection of the abdomen and lower extremities, the surgeon made two small incisions of about $5 \mathrm{~mm}$ around the umbilical cord and obtained about 100 to $150 \mathrm{ml}$ of adipose tissue from the subcutaneous tissue around the umbilicus using the superwet technique. Thereafter, sutures were used to close the wound, whereas the abdominal binder was used to ensure that the bruises do not occur in the surgical area. Finally, we stored the harvested adipose tissue in a sterile container, followed by packaging it in a portable cryopreservation box. This was done so that the collected adipose tissue would not be damaged on the way to the laboratory.

\section{SVF isolation and injection}

Briefly, lipoaspirates were washed with phosphate-buffered saline, while the mesh filter was applied to remove containing residual blood cells and tissue fragments. Next, an equal volume of digestive enzyme (type I collagenase; Worthington, Lakewood, NJ, USA) was mixed with the washed adipose tissue and placed in a shaking incubator at $37^{\circ} \mathrm{C}$ for 30 minutes. The resulting mixture was then centrifuged at a rate of $1000 \mathrm{~g}$ for 10 minutes and subsequently discarded the supernatant (Eppendorf 5810R, Germany). After this, the remnant SVF at the bottom was resuspended in PBS up to a volume of 4.5-ml SVF, whereas an automatic cell counter (Countstar IC1000, China) was used to quantify cell quantity and viability.

In short, about $4 \mathrm{ml}$ of SVF suspension was injected into the region of the cartilage defect by a trained experienced orthopedic surgeon. Upon the injection of SVF, subcuticular suture and pressure dressing were performed. All the operations were performed by the same experienced orthopedic surgeon.

\section{Statistical analysis}

Changes in all follow-up data including VAS, WOMAC, WORMS, and MOCART, among others, were determined using a paired t-test. The discrete data were analyzed by chi-square test. A value of $p<0.05$ was considered statistically significant. Data displayed in the graphs are means with standard deviation. All statistical data analysis was executed using SPSS software (version 20.0, IBM Corporation, NY, US).

\section{Results}

\section{Patient profiles}

The flowchart of the clinical trial is depicted in Fig. 3. Among the 131 patients screened for the study, only 47 patients (29 women and 18 men) were eligible based on clinical examination and MRI. Overall, 53 knees (47 patients) with osteoarthritis received an intra-articular injection of SVF. We enrolled patients between January 2018 and January 2020, where the last patient completed 1-year follow-up in January 2021. Further details on patient characteristics showed in Table 1. 
Table 1

Study participant demographic characteristics

\begin{tabular}{|ll|}
\hline Characteristics & Baseline Data \\
\hline Age; years & $50.83 \pm 10.88$ \\
\hline Sex (M/F); n (\%) & $18 / 29(38 \% / 62 \%)$ \\
\hline Knee (R/L); n (\%) & $30 / 23(57 \% / 43 \%)$ \\
\hline BMl; kg/m² & $22.67 \pm 3.68$ \\
\hline K- L classification (\%) & $0(0 \%)$ \\
I & \\
\hline II & $29(55 \%)$ \\
\hline III & $24(45 \%)$ \\
\hline IV & $0(0 \%)$ \\
\hline M male; F female; R right; L left; BMI body mass index \\
\hline
\end{tabular}

\section{Adverse effects}

During the follow-up period, we observed no serious adverse events such as infection, allergy, and poor wound healing. However, the most common adverse events were pain and swelling of the knee in the postoperative, which occurred in 6 patients (13\%). Notably, Celebrex exhibited a significant effect on relieving the pain of the knee joint. We noted that all pain and swelling of the knee joint resolved within two weeks.

\section{Clinical outcome}

After one year of follow-up, the VAS, WOMAC pain, stiffness, and physical function of the patients were evaluated at baseline, 1, 3, 6, and 12 months after injection with SVF (Table 2). The mean WOMAC pain, stiffness, and physical function scores decreased from $9.38 \pm 0.96$ to $2.69 \pm 1.02$ (71\% decrease; $p<0.001)$, from $2.83 \pm 0.75$ to $0.93 \pm 0.74$ (67\% decrease; $p$ $<0.001)$ and from $24.66 \pm 3.12$ to $10.14 \pm 2.24(59 \%$ decrease; $p<0.001)$ in the patients with grade $20 A$, while those of patients with grade $3 \mathrm{OA}$ also decreased from $12.42 \pm 1.53$ to $4.92 \pm 1.22$ ( $60 \%$ decrease; $p<0.001$ ), from $4.88 \pm 1.17$ to 2.41 \pm 1.35 ( $51 \%$ decrease; $p<0.001$ ), and from $33.71 \pm 4.51$ to $17.58 \pm 4.35$ ( $48 \%$ decrease; $p<0.001)$, respectively, between baseline and last follow-up (Fig. 4a, b, c). The mean VAS scores improved from $4.31 \pm 0.46$ to $1.59 \pm 0.93(63 \%$ decrease; $p<$ $0.001)$ in OA grade 2 ; from $6.04 \pm 0.61$ to $2.88 \pm 0.78$ ( $52 \%$ decrease; $p<0.001)$ in OA grade 3 , which showed a statistically significant difference (Fig. 4d). 
The results of clinical evaluation

\begin{tabular}{|c|c|c|c|c|}
\hline & \multicolumn{2}{|l|}{ Grade 2} & \multicolumn{2}{|l|}{ Grade 3} \\
\hline & Mean \pm SD & $P$ value & Mean \pm SD & $P$ value \\
\hline \multicolumn{5}{|c|}{ WOMAC pain } \\
\hline Baseline & $9.38 \pm 0.96$ & & $12.42 \pm 1.53$ & \\
\hline 1 month & $6.62 \pm 0.93$ & $<0.001$ & $9.88 \pm 1.45$ & $<0.001$ \\
\hline 3 months & $4.10 \pm 0.99$ & $<0.001$ & $6.58 \pm 1.13$ & $<0.001$ \\
\hline 6 months & $3.24 \pm 0.77$ & $<0.001$ & $5.50 \pm 1.16$ & $<0.001$ \\
\hline 12 months & $2.69 \pm 1.02$ & $<0.001$ & $4.92 \pm 1.22$ & $<0.001$ \\
\hline \multicolumn{5}{|c|}{ WOMAC stiffness } \\
\hline Baseline & $2.83 \pm 0.75$ & & $4.88 \pm 1.17$ & \\
\hline 1 month & $1.72 \pm 0.69$ & $<0.001$ & $3.33 \pm 1.31$ & $<0.001$ \\
\hline 3 months & $1.21 \pm 0.61$ & $<0.001$ & $2.63 \pm 1.28$ & $<0.001$ \\
\hline 6 months & $0.86 \pm 0.68$ & $<0.001$ & $2.17 \pm 1.25$ & $<0.001$ \\
\hline 12 months & $0.93 \pm 0.74$ & $<0.001$ & $2.41 \pm 1.35$ & $<0.001$ \\
\hline \multicolumn{5}{|c|}{ WOMAC physical function } \\
\hline Baseline & $24.66 \pm 3.12$ & & $33.71 \pm 4.51$ & \\
\hline 1 month & $18.69 \pm 3.11$ & $<0.001$ & $27.83 \pm 4.51$ & $<0.001$ \\
\hline 3 months & $13.03 \pm 2.23$ & $<0.001$ & $20.13 \pm 4.23$ & $<0.001$ \\
\hline 6 months & $11.17 \pm 2.23$ & $<0.001$ & $18.04 \pm 3.75$ & $<0.001$ \\
\hline 12 months & $10.14 \pm 2.24$ & $<0.001$ & $17.58 \pm 4.35$ & $<0.001$ \\
\hline \multicolumn{5}{|l|}{ VAS score } \\
\hline Baseline & $4.31 \pm 0.46$ & & $6.04 \pm 0.61$ & \\
\hline 1 month & $2.90 \pm 0.66$ & $<0.001$ & $4.21 \pm 0.71$ & $<0.001$ \\
\hline 3 months & $2.07 \pm 0.91$ & $<0.001$ & $3.25 \pm 0.78$ & $<0.001$ \\
\hline 6 months & $1.41 \pm 0.81$ & $<0.001$ & $2.67 \pm 0.80$ & $<0.001$ \\
\hline 12 months & $1.59 \pm 0.93$ & $<0.001$ & $2.88 \pm 0.78$ & $<0.001$ \\
\hline \multicolumn{5}{|l|}{ Knee ROM } \\
\hline Baseline & $123.72 \pm 3.44$ & & $114.21 \pm 5.97$ & \\
\hline 1 month & $132.41 \pm 4.24$ & $<0.001$ & $124.67 \pm 5.72$ & $<0.001$ \\
\hline 3 months & $136.38 \pm 3.68$ & $<0.001$ & $130.08 \pm 5.32$ & $<0.001$ \\
\hline 6 months & $137.69 \pm 3.26$ & $<0.001$ & $130.88 \pm 5.33$ & $<0.001$ \\
\hline 12 months & $137.82 \pm 3.44$ & $<0.001$ & $130.62 \pm 5.72$ & $<0.001$ \\
\hline
\end{tabular}


For patients with $\mathrm{K}-\mathrm{L}$ grade 2 , the mean ROM increased from a baseline of $123.72 \pm 3.44^{\circ}$ to $132.41 \pm 4.24^{\circ}$ at 1 month, 3 months $\left(136.38 \pm 3.68^{\circ}\right), 6$ months $\left(137.69 \pm 3.26^{\circ}\right)$, and 12 months $\left(137.82 \pm 3.44^{\circ}\right)$. Similarly, for patients with $\mathrm{K}-\mathrm{L}$ grade 3 , the mean ROM improved from a baseline of $114.21 \pm 5.97^{\circ}$ to $124.67 \pm 5.72^{\circ}$ at 1 month, 3 months $\left(130.08 \pm 5.32^{\circ}\right), 6$ months $\left(130.88 \pm 5.33^{\circ}\right)$, and 12 months $\left(130.62 \pm 5.72^{\circ}\right.$ ) (Fig. 4e). We identified that the greatest improvement occurred during the first and third months, which corresponded with our results of WOMAC and VAS scores. Afterward, no marked increase was observed in OA grade 2 and 3. Despite this, these results demonstrated that intra-articular injection of SVF can effectively improve the quality of life of patients with $O A$.

\section{Radiological outcomes}

Following SVF injection, 47 patients completed MRI evaluation at baseline, 6, and 12 months (Fig. 5). We subsequently found no signs of new cyst formation, neoplasms of the bone, cartilage, and synovium. The WORMS scores based on MRI used for the whole-organ assessment of the knee at baseline, 6, and 12 months, are presented in Table 3. We observed that the mean WORMS score improved from a baseline of $54.86 \pm 8.15$ to $40.48 \pm 7.28$ ( $26 \%$ decrease; $p<0.001)$ at 12 months, in patients with grade $2 \mathrm{OA}$. Likewise, in patients with grade $3 \mathrm{OA}$, the WORMS score revealed a significant improvement. The mean scores decreased from a baseline of $75.67 \pm 10.44$ to $57.46 \pm 8.03(24 \%$ decrease; $p<0.001)$ at 12 months.

Table 3

WORMS changes during 12-months follow-up

\begin{tabular}{|c|c|c|c|c|c|c|c|c|c|c|}
\hline \multirow[t]{2}{*}{ Variables } & \multicolumn{5}{|l|}{ Grade 2} & \multicolumn{5}{|l|}{ Grade 3} \\
\hline & Baseline & $\begin{array}{l}6 \\
\text { months }\end{array}$ & $\begin{array}{l}P \\
\text { value }\end{array}$ & $\begin{array}{l}12 \\
\text { months }\end{array}$ & $\begin{array}{l}P \\
\text { value }\end{array}$ & Baseline & $\begin{array}{l}6 \\
\text { months }\end{array}$ & $\begin{array}{l}P \\
\text { value }\end{array}$ & $\begin{array}{l}12 \\
\text { months }\end{array}$ & $\begin{array}{l}P \\
\text { value }\end{array}$ \\
\hline Cartilage & $\begin{array}{l}26.48 \pm \\
3.43\end{array}$ & $\begin{array}{l}19.38 \\
\pm 2.91\end{array}$ & $\dot{0} 001$ & $\begin{array}{l}15.17 \\
\pm 2.96\end{array}$ & $\dot{0} .001$ & $\begin{array}{l}34.33 \pm \\
5.89\end{array}$ & $\begin{array}{l}25.75 \\
\pm 4.39\end{array}$ & $\dot{0} 001$ & $\begin{array}{l}19.58 \\
\pm 3.83\end{array}$ & $<$. \\
\hline $\begin{array}{l}\text { Marrow } \\
\text { abnormality }\end{array}$ & $\begin{array}{l}3.07 \pm \\
1.01\end{array}$ & $\begin{array}{l}1.97 \pm \\
0.96\end{array}$ & $<.001$ & $\begin{array}{l}1.72 \pm \\
0.91\end{array}$ & $<.001$ & $\begin{array}{l}4.42 \pm \\
1.11\end{array}$ & $\begin{array}{l}3.25 \pm \\
0.88\end{array}$ & $\dot{0} 001$ & $\begin{array}{l}2.88 \pm \\
0.60\end{array}$ & $\dot{0.001}$ \\
\hline Bone cysts & $\begin{array}{l}2.31 \pm \\
1.02\end{array}$ & $\begin{array}{l}1.76 \pm \\
0.94\end{array}$ & $\begin{array}{l}< \\
0.05\end{array}$ & $\begin{array}{l}1.69 \pm \\
0.91\end{array}$ & $\begin{array}{l}<.05 \\
0.05\end{array}$ & $\begin{array}{l}3.71 \pm \\
0.68\end{array}$ & $\begin{array}{l}3.04 \pm \\
0.68\end{array}$ & $\begin{array}{l}< \\
0.05\end{array}$ & $\begin{array}{l}2.91 \pm \\
0.70\end{array}$ & $<$. \\
\hline $\begin{array}{l}\text { Bone } \\
\text { attrition }\end{array}$ & $\begin{array}{l}1.03 \pm \\
0.93\end{array}$ & $\begin{array}{l}0.90 \pm \\
0.80\end{array}$ & 0.535 & $\begin{array}{l}0.83 \pm \\
0.75\end{array}$ & 0.353 & $\begin{array}{l}2.50 \pm \\
0.65\end{array}$ & $\begin{array}{l}2.38 \pm \\
0.63\end{array}$ & 0.500 & $\begin{array}{l}2.25 \pm \\
0.60\end{array}$ & 0.179 \\
\hline Osteophytes & $\begin{array}{l}19.97 \pm \\
3.99\end{array}$ & $\begin{array}{l}19.69 \\
\pm 4.07\end{array}$ & 0.799 & $\begin{array}{l}19.59 \\
\pm 4.03\end{array}$ & 0.726 & $\begin{array}{l}27.08 \pm \\
4.75\end{array}$ & $\begin{array}{l}26.67 \\
\pm 4.76\end{array}$ & 0.766 & $\begin{array}{l}26.63 \\
\pm 4.68\end{array}$ & 0.743 \\
\hline Menisci & $\begin{array}{l}0.83 \pm \\
1.12\end{array}$ & $\begin{array}{l}0.59 \pm \\
0.81\end{array}$ & 0.325 & $\begin{array}{l}0.55 \pm \\
0.77\end{array}$ & 0.261 & $\begin{array}{l}1.67 \pm \\
1.28\end{array}$ & $\begin{array}{l}1.50 \pm \\
1.08\end{array}$ & 0.628 & $\begin{array}{l}1.54 \pm \\
1.18\end{array}$ & 0.716 \\
\hline Ligaments & $\begin{array}{l}0.07 \pm \\
0.25\end{array}$ & $\begin{array}{l}0.03 \pm \\
0.18\end{array}$ & 0.538 & $\begin{array}{l}0.03 \pm \\
0.18\end{array}$ & 0.538 & $\begin{array}{l}0.17 \pm \\
0.37\end{array}$ & $\begin{array}{l}0.13 \pm \\
0.33\end{array}$ & 0.669 & $\begin{array}{l}0.08 \pm \\
0.28\end{array}$ & 0.393 \\
\hline Synovitis & $\begin{array}{l}1.10 \pm \\
0.71\end{array}$ & $\begin{array}{l}0.93 \pm \\
0.74\end{array}$ & 0.374 & $\begin{array}{l}0.90 \pm \\
0.71\end{array}$ & 0.286 & $\begin{array}{l}1.79 \pm \\
0.64\end{array}$ & $\begin{array}{l}1.63 \pm \\
0.75\end{array}$ & 0.436 & $\begin{array}{l}1.58 \pm \\
0.76\end{array}$ & 0.330 \\
\hline $\begin{array}{l}\text { WORMS } \\
\text { total }\end{array}$ & $\begin{array}{l}54.86 \pm \\
8.15\end{array}$ & $\begin{array}{l}45.24 \\
\pm 7.52\end{array}$ & $\begin{array}{l}< \\
0.001\end{array}$ & $\begin{array}{l}40.48 \\
\pm 7.28\end{array}$ & $\begin{array}{l}< \\
0.001\end{array}$ & $\begin{array}{l}75.67 \pm \\
10.44\end{array}$ & $\begin{array}{l}64.33 \\
\pm 9.09\end{array}$ & $\begin{array}{l}< \\
0.001\end{array}$ & $\begin{array}{l}57.46 \\
\pm 8.03\end{array}$ & $\begin{array}{l}< \\
0.001\end{array}$ \\
\hline
\end{tabular}

The repair of the cartilage defects was measured using the MOCART system at 6 and 12 months (Table 4). The mean MOCART score improved from $52.93 \pm 13.87$ to and $62.07 \pm 12.83$ at 6 and 12 months, respectively, in patients with K-L grade 2. Similarly, it increased from $46.46 \pm 10.05$ to $57.08 \pm 11.98$ at 6 and 12 months in patients with K-L grade 3 , respectively. In addition, there were 12 knees $(41.38 \%)$ that showed complete or hypertrophic repair tissue filling of the defect in OA grade 2 , while 13 knees (44.83\%) elucidated most of the repair of cartilage defects. Finally, although only one knee (4.17\%) showed complete repair of the cartilage defects in grade OA 3, there were 18 knees (75.00\%) that showed substantial repair of cartilage defects. 


\begin{tabular}{|c|c|c|c|c|c|}
\hline \multirow[t]{2}{*}{ Variables } & \multirow{2}{*}{$\begin{array}{l}\text { Maximum } \\
\text { score }\end{array}$} & \multicolumn{2}{|l|}{ Grade 2} & \multicolumn{2}{|l|}{ Grade 3} \\
\hline & & 6 months & 12 months & 6 months & 12 months \\
\hline \multicolumn{6}{|l|}{$\begin{array}{l}\text { 1. Degree of defect repair and filling of the } \\
\text { defect }\end{array}$} \\
\hline Complete & 20 & $0(0)$ & $2(6.90)$ & $0(0)$ & $1(4.17)$ \\
\hline Hypertrophy & 15 & $9(31.03)$ & $10(34.48)$ & $5(20.83)$ & $8(33.33)$ \\
\hline \multicolumn{6}{|l|}{ Incomplete } \\
\hline$>50 \%$ of the adjacent cartilage & 10 & $12(41.38)$ & $13(44.83)$ & $9(37.50)$ & $10(41.66)$ \\
\hline$<50 \%$ of the adjacent cartilage & 5 & $7(21.14)$ & $4(13.79)$ & $7(29.17)$ & $4(16.67)$ \\
\hline subchondral bone exposed & 0 & $1(3.45)$ & $0(0)$ & $3(12.50)$ & $1(4.17)$ \\
\hline \multicolumn{6}{|l|}{ 2. Integration to border zone } \\
\hline Complete & 15 & $7(21.14)$ & $14(48.28)$ & $4(16.67)$ & $7(29.16)$ \\
\hline \multicolumn{6}{|l|}{ Incomplete } \\
\hline Demarcating border visible (split-like) & 10 & $17(58.62)$ & $12(41.38)$ & $10(41.67)$ & $9(37.50)$ \\
\hline \multicolumn{6}{|l|}{ Defect visible } \\
\hline$<50 \%$ of length of the repair tissue & 5 & $3(10.34)$ & $3(10.34)$ & $7(29.17)$ & $6(25.00)$ \\
\hline$>50 \%$ of length of the repair tissue & 0 & $2(6.90)$ & $0(0)$ & $3(12.50)$ & $2(8.33)$ \\
\hline \multicolumn{6}{|l|}{ 3. Surface of the repair tissue } \\
\hline Surface intact & 10 & $16(55.17)$ & $19(65.52)$ & $13(54.17)$ & $16(66.67)$ \\
\hline \multicolumn{6}{|l|}{ Surface damaged } \\
\hline$<50 \%$ of repair tissue depth & 5 & $11(37.93)$ & $10(34.48)$ & $9(37.50)$ & $7(29.16)$ \\
\hline $\begin{array}{l}>50 \% \text { of repair tissue depth or total } \\
\text { degeneration }\end{array}$ & 0 & $2(6.90)$ & $0(0)$ & $2(8.33)$ & $1(4.17)$ \\
\hline \multicolumn{6}{|l|}{ 4. Structure of the repair tissue } \\
\hline Homogeneous & 5 & $20(68.97)$ & $23(79.31)$ & $16(66.67)$ & 19 (79.17) \\
\hline Inhomogeneous or cleft formation & 0 & $9(31.03)$ & $6(20.69)$ & $8(33.33)$ & $5(20.83)$ \\
\hline \multicolumn{6}{|l|}{ 5. Signal intensity of repair tissue } \\
\hline Normal (identical to adjacent cartilage) & 30 & $2(6.90)$ & $7(24.14)$ & $1(4.17)$ & $3(12.50)$ \\
\hline $\begin{array}{l}\text { Nearly normal (slight areas of signal } \\
\text { alteration) }\end{array}$ & 15 & $20(68.96)$ & $18(62.07)$ & $16(66.67)$ & $17(70.83)$ \\
\hline Abnormal (large areas of signal alteration) & 0 & $7(21.14)$ & $4(13.79)$ & $7(29.16)$ & $4(16.67)$ \\
\hline \multicolumn{6}{|l|}{ 6. Subchondral lamina } \\
\hline Intact & 5 & $21(72.41)$ & $21(72.41)$ & $17(70.83)$ & $20(83.33)$ \\
\hline Not intact & 0 & $8(27.59)$ & $8(27.59)$ & $7(29.17)$ & $4(16.67)$ \\
\hline
\end{tabular}




\begin{tabular}{|lccccc|}
\hline Variables & $\begin{array}{l}\text { Maximum } \\
\text { score }\end{array}$ & Grade 2 & & Grade 3 \\
\cline { 3 - 5 } & & $\mathbf{6}$ months & $\mathbf{1 2}$ months & $\mathbf{6}$ months & $\mathbf{1 2}$ months \\
\hline Intact & 5 & $8(27.59)$ & $8(27.59)$ & $8(33.33)$ & $11(45.83)$ \\
\hline $\begin{array}{l}\text { Not intact (edema, granulation tissue, cysts, } \\
\text { sclerosis) }\end{array}$ & 0 & $21(72.41)$ & $21(72.41)$ & $16(66.67)$ & $13(54.17)$ \\
\hline 8. Adhesions & & & & & \\
\hline No & 5 & $18(62.07)$ & $18(62.07)$ & $12(50.00)$ & $16(66.67)$ \\
\hline Yes & 0 & $11(37.93)$ & $11(37.93)$ & $12(50.00)$ & $8(33.33)$ \\
\hline 9. Synovitis & & & & \\
\hline No synovitis & 5 & $9(31.03)$ & $9(31.03)$ & $2(8.33)$ & $4(16.67)$ \\
\hline Synovitis & 0 & $20(68.97)$ & $20(68.97)$ & $22(91.67)$ & $20(83.33)$ \\
\hline Mean \pm SD & & $52.93 \pm$ & $62.07 \pm$ & $46.46 \pm$ & $57.08 \pm$ \\
\hline
\end{tabular}

\section{Changes in parameters of 3D cartilage model}

To establish the 3D cartilage model, all patients finished the examination of 3D-FS-SPGR sequence at baseline, 6 , and 12 months. Our findings revealed that the parameters of 3D cartilage model improved both in patients with OA K-L grades 2 and 3 (Table 5 and Fig. 6). In patients with K-L grade 2, the thickness of cartilage defect decreased from $1.53 \pm 0.23 \mathrm{~mm}$ to $0.92 \pm$ $0.18 \mathrm{~mm}$ in MF (40\% decrease; $p<0.001)$; from $1.46 \pm 0.30 \mathrm{~mm}$ to $1.17 \pm 0.26$ in LF $(20 \%$ decrease; $p<0.001)$; from $1.45 \pm$ $0.25 \mathrm{~mm}$ to $1.25 \pm 0.21 \mathrm{~mm}$ in T ( $14 \%$ decrease; $p<0.05$ ); from $1.43 \pm 0.26 \mathrm{~mm}$ to $0.96 \pm 0.22 \mathrm{~mm}$ in MT (33\% decrease; $p<$ 0.001 ); from $1.34 \pm 0.19 \mathrm{~mm}$ to $1.13 \pm 0.18 \mathrm{~mm}$ in $\mathrm{LT}$ ( $16 \%$ decrease; $p<0.001$ ); and from $1.29 \pm 0.19 \mathrm{~mm}$ to $1.01 \pm 0.15 \mathrm{~mm}$ in $\mathrm{P}(22 \%$ decrease; $\mathrm{p}<0.001)$. The volume of cartilage defect decreased by $84.00 \pm 32.30 \mathrm{~mm}^{3}$ in $\mathrm{MF}(52 \%$ decrease; $\mathrm{p}<$ $0.001)$; by $94.73 \pm 45.55 \mathrm{~mm}^{3}$ in LF ( $35 \%$ decrease; $\left.p<0.001\right)$; by $64.18 \pm 21.40 \mathrm{~mm}^{3}$ in MT $(54 \%$ decrease; $p<0.001)$; by $88.66 \pm 28.04 \mathrm{~mm}^{3}$ in LT (26\% decrease; $\left.p<0.001\right)$; but not in T and $\mathrm{P}$, from $147.91 \pm 61.35 \mathrm{~mm}^{3}$ to $112.80 \pm 56.09 \mathrm{~mm}^{3}$ in $\mathrm{T}$ ( $24 \%$ decrease; $p=0.085)$; from $137.29 \pm 53.30 \mathrm{~mm}^{3}$ to $102.15 \pm 43.47 \mathrm{~mm}^{3}$ in $\mathrm{P}(26 \%$ decrease; $p=0.095)$. We further found that the surface of cartilage defect decreased in MF, LF, T, MT, and LT, showing a statistically significant difference. Nevertheless, we observed no statistical difference in $P(27 \%$ decrease; $p=0.057)$. As for the healthy cartilage, we generally identified no significe difference, except for MT and LT. Subsequently, we noted that the volume of healthy cartilage increased from $1647.92 \pm 200.24 \mathrm{~mm}^{3}$ to $1783.31 \pm 202.94 \mathrm{~mm}^{3}$ and from $1613.65 \pm 147.04 \mathrm{~mm}^{3}$ to $1694.24 \pm 150.56 \mathrm{~mm}^{3}$ in MT $(8 \%$ increase; $p<0.05)$ and LT $(5 \%$ increase; $p<0.05)$, respectively. 
Table 5

Changes in cartilage model

\begin{tabular}{|c|c|c|c|c|c|c|c|c|}
\hline & $\begin{array}{l}\text { Volume of } \\
\text { defective cartilage } \\
\left(\mathrm{mm}^{3}\right)\end{array}$ & $\begin{array}{l}P \\
\text { value }\end{array}$ & $\begin{array}{l}\text { Size of defective } \\
\text { cartilage }\left(\mathrm{mm}^{2}\right)\end{array}$ & $\begin{array}{l}P \\
\text { value }\end{array}$ & $\begin{array}{l}\text { Volume of } \\
\text { healthy cartilage } \\
\left(\mathrm{mm}^{3}\right)\end{array}$ & $\begin{array}{l}P \\
\text { value }\end{array}$ & $\begin{array}{l}\text { Thickness } \\
\text { of } \\
\text { defective } \\
\text { cartilage } \\
(\mathrm{mm})\end{array}$ & $\begin{array}{l}\mathrm{P} \\
\text { value }\end{array}$ \\
\hline \multicolumn{9}{|l|}{ Grade 2} \\
\hline \multicolumn{9}{|l|}{ MF } \\
\hline Baseline & $173.82 \pm 63.41$ & & $353.86 \pm 122.99$ & & $\begin{array}{l}3102.37 \pm \\
435.02\end{array}$ & & $\begin{array}{l}1.53 \pm \\
0.23\end{array}$ & \\
\hline $\begin{array}{l}6 \\
\text { months }\end{array}$ & $123.13 \pm 46.87$ & $<.001$ & $257.17 \pm 95.64$ & $<.001$ & $\begin{array}{l}3231.87 \pm \\
451.13\end{array}$ & 0.279 & $\begin{array}{l}1.16 \pm \\
0.20\end{array}$ & <. 001 \\
\hline $\begin{array}{l}12 \\
\text { months }\end{array}$ & $84.00 \pm 32.30$ & $\dot{0} 001$ & $182.22 \pm 67.00$ & $\dot{0} 001$ & $\begin{array}{l}3317.69 \pm \\
447.02\end{array}$ & 0.073 & $\begin{array}{l}0.92 \pm \\
0.18\end{array}$ & $\begin{array}{l}<.001 \\
0.00\end{array}$ \\
\hline \multicolumn{9}{|l|}{ LF } \\
\hline Baseline & $146.10 \pm 61.17$ & & $302.77 \pm 101.75$ & & $\begin{array}{l}3070.04 \pm \\
428.12\end{array}$ & & $\begin{array}{l}1.46 \pm \\
0.30\end{array}$ & \\
\hline $\begin{array}{l}6 \\
\text { months }\end{array}$ & $116.49 \pm 51.34$ & $\hat{0}_{0.05}$ & $244.22 \pm 96.33$ & $\begin{array}{l}<.05 \\
0.05\end{array}$ & $\begin{array}{l}3116.65 \pm \\
422.88\end{array}$ & 0.557 & $\begin{array}{l}1.25 \pm \\
0.27\end{array}$ & $<.05$ \\
\hline $\begin{array}{l}12 \\
\text { months }\end{array}$ & $94.73 \pm 45.55$ & $<.001$ & $199.93 \pm 86.07$ & $\begin{array}{l}<.001 \\
0.00\end{array}$ & $\begin{array}{l}3179.09 \pm \\
426.00\end{array}$ & 0.343 & $\begin{array}{l}1.17 \pm \\
0.26\end{array}$ & $\begin{array}{l}<.001 \\
0.00\end{array}$ \\
\hline \multicolumn{9}{|l|}{$\mathrm{T}$} \\
\hline Baseline & $147.91 \pm 61.35$ & & $309.72 \pm 99.22$ & & $\begin{array}{l}2568.48 \pm \\
406.67\end{array}$ & & $\begin{array}{l}1.45 \pm \\
0.25\end{array}$ & \\
\hline $\begin{array}{l}6 \\
\text { months }\end{array}$ & $127.76 \pm 57.33$ & 0.318 & $262.86 \pm 97.90$ & 0.172 & $\begin{array}{l}2617.60 \pm \\
408.53\end{array}$ & 0.645 & $\begin{array}{l}1.34 \pm \\
0.23\end{array}$ & 0.153 \\
\hline $\begin{array}{l}12 \\
\text { months }\end{array}$ & $112.80 \pm 56.09$ & 0.085 & $222.52 \pm 98.57$ & $\begin{array}{l}< \\
0.05\end{array}$ & $\begin{array}{l}2658.51 \pm \\
410.85\end{array}$ & 0.412 & $\begin{array}{l}1.25 \pm \\
0.21\end{array}$ & $\begin{array}{l}<.05 \\
0.0\end{array}$ \\
\hline \multicolumn{9}{|l|}{ MT } \\
\hline Baseline & $139.72 \pm 46.15$ & & $281.79 \pm 80.48$ & & $\begin{array}{l}1647.92 \pm \\
200.24\end{array}$ & & $\begin{array}{l}1.43 \pm \\
0.26\end{array}$ & \\
\hline $\begin{array}{l}6 \\
\text { months }\end{array}$ & $95.43 \pm 31.56$ & $<.001$ & $206.20 \pm 63.30$ & $<.001$ & $\begin{array}{l}1720.68 \pm \\
197.61\end{array}$ & 0.178 & $\begin{array}{l}1.15 \pm \\
0.23\end{array}$ & $<.001$ \\
\hline $\begin{array}{l}12 \\
\text { months }\end{array}$ & $64.18 \pm 21.40$ & $<.001$ & $146.15 \pm 45.47$ & $\begin{array}{l}<.001 \\
0.001\end{array}$ & $\begin{array}{l}1783.31 \pm \\
202.94\end{array}$ & $<.05$ & $\begin{array}{l}0.96 \pm \\
0.22\end{array}$ & $\begin{array}{l}<.001 \\
\end{array}$ \\
\hline \multicolumn{9}{|l|}{ LT } \\
\hline Baseline & $119.87 \pm 32.51$ & & $256.78 \pm 64.51$ & & $\begin{array}{l}1613.65 \pm \\
147.04\end{array}$ & & $\begin{array}{l}1.34 \pm \\
0.19\end{array}$ & \\
\hline $\begin{array}{l}6 \\
\text { months }\end{array}$ & $101.62 \pm 30.18$ & 0.055 & $209.44 \pm 56.13$ & $\hat{0}_{0.05}$ & $\begin{array}{l}1656.77 \pm \\
150.76\end{array}$ & 0.284 & $\begin{array}{l}1.22 \pm \\
0.19\end{array}$ & $<.05$ \\
\hline $\begin{array}{l}12 \\
\text { months }\end{array}$ & $88.66 \pm 28.04$ & $\begin{array}{l}< \\
0.05\end{array}$ & $178.79 \pm 54.55$ & $\begin{array}{l}< \\
0.001\end{array}$ & $\begin{array}{l}1694.24 \pm \\
150.56\end{array}$ & $\begin{array}{l}< \\
0.05\end{array}$ & $\begin{array}{l}1.13 \pm \\
0.18\end{array}$ & $\begin{array}{l}<.001 \\
0.00\end{array}$ \\
\hline
\end{tabular}




\begin{tabular}{|c|c|c|c|c|c|c|c|c|}
\hline & $\begin{array}{l}\text { Volume of } \\
\text { defective cartilage } \\
\left(\mathrm{mm}^{3}\right)\end{array}$ & $\begin{array}{l}P \\
\text { value }\end{array}$ & $\begin{array}{l}\text { Size of defective } \\
\text { cartilage }\left(\mathrm{mm}^{2}\right)\end{array}$ & $\begin{array}{l}P \\
\text { value }\end{array}$ & $\begin{array}{l}\text { Volume of } \\
\text { healthy cartilage } \\
\left(\mathrm{mm}^{3}\right)\end{array}$ & $\begin{array}{l}P \\
\text { value }\end{array}$ & $\begin{array}{l}\text { Thickness } \\
\text { of } \\
\text { defective } \\
\text { cartilage } \\
(\mathrm{mm})\end{array}$ & $\begin{array}{l}P \\
\text { value }\end{array}$ \\
\hline Baseline & $137.29 \pm 53.30$ & & $292.45 \pm 106.74$ & & $\begin{array}{l}2304.81 \pm \\
181.21\end{array}$ & & $\begin{array}{l}1.29 \pm \\
0.19\end{array}$ & \\
\hline $\begin{array}{l}6 \\
\text { months }\end{array}$ & $117.78 \pm 46.70$ & 0.347 & $247.55 \pm 89.12$ & 0.268 & $\begin{array}{l}2354.98 \pm \\
182.95\end{array}$ & 0.304 & $\begin{array}{l}1.10 \pm \\
0.16\end{array}$ & $\begin{array}{l}<.05 \\
0.05\end{array}$ \\
\hline $\begin{array}{l}12 \\
\text { months }\end{array}$ & $102.15 \pm 43.47$ & 0.095 & $213.88 \pm 82.64$ & 0.057 & $\begin{array}{l}2394.72 \pm \\
180.11\end{array}$ & 0.067 & $\begin{array}{l}1.01 \pm \\
0.15\end{array}$ & $\begin{array}{l}< \\
0.001\end{array}$ \\
\hline \multicolumn{9}{|l|}{ Grade 3} \\
\hline \multicolumn{9}{|l|}{ MF } \\
\hline Baseline & $278.10 \pm 110.58$ & & $525.43 \pm 167.38$ & & $\begin{array}{l}2382.20 \pm \\
314.39\end{array}$ & & $\begin{array}{l}1.72 \pm \\
0.32\end{array}$ & \\
\hline $\begin{array}{l}6 \\
\text { months }\end{array}$ & $198.80 \pm 79.19$ & $\begin{array}{l}< \\
0.05\end{array}$ & $408.84 \pm 144.89$ & $\begin{array}{l}< \\
0.05\end{array}$ & $\begin{array}{l}2540.67 \pm \\
323.21\end{array}$ & 0.105 & $\begin{array}{l}1.34 \pm \\
0.25\end{array}$ & $\begin{array}{l}< \\
0.001\end{array}$ \\
\hline $\begin{array}{l}12 \\
\text { months }\end{array}$ & $130.30 \pm 49.56$ & $<0.001$ & $286.18 \pm 108.47$ & $<0.001$ & $\begin{array}{l}2712.22 \pm \\
343.55\end{array}$ & $\begin{array}{l}<.05 \\
0.05\end{array}$ & $\begin{array}{l}1.03 \pm \\
0.23\end{array}$ & $\begin{array}{l}< \\
0.001\end{array}$ \\
\hline \multicolumn{9}{|l|}{ LF } \\
\hline Baseline & $229.23 \pm 94.05$ & & $459.71 \pm 176.88$ & & $\begin{array}{l}2379.37 \pm \\
235.44\end{array}$ & & $\begin{array}{l}1.74 \pm \\
0.28\end{array}$ & \\
\hline $\begin{array}{l}6 \\
\text { months }\end{array}$ & $190.17 \pm 79.75$ & 0.111 & $390.81 \pm 153.97$ & 0.144 & $\begin{array}{l}2472.52 \pm \\
270.39\end{array}$ & 0.241 & $\begin{array}{l}1.53 \pm \\
0.25\end{array}$ & $\begin{array}{l}< \\
0.05\end{array}$ \\
\hline $\begin{array}{l}12 \\
\text { months }\end{array}$ & $162.17 \pm 70.92$ & $\begin{array}{l}<.05 \\
0.05\end{array}$ & $339.47 \pm 144.43$ & $\begin{array}{l}<.05 \\
0.05\end{array}$ & $\begin{array}{l}2562.15 \pm \\
276.73\end{array}$ & $\begin{array}{l}<.05 \\
0.05\end{array}$ & $\begin{array}{l}1.36 \pm \\
0.23\end{array}$ & $<.001$ \\
\hline \multicolumn{9}{|l|}{$\mathrm{T}$} \\
\hline Baseline & $196.75 \pm 77.85$ & & $410.31 \pm 152.60$ & & $\begin{array}{l}2190.18 \pm \\
198.06\end{array}$ & & $\begin{array}{l}1.55 \pm \\
0.30\end{array}$ & \\
\hline $\begin{array}{l}6 \\
\text { months }\end{array}$ & $166.80 \pm 69.83$ & 0.179 & $352.21 \pm 139.03$ & 0.189 & $\begin{array}{l}2261.72 \pm \\
210.30\end{array}$ & 0.256 & $\begin{array}{l}1.34 \pm \\
0.28\end{array}$ & $\begin{array}{l}< \\
0.05\end{array}$ \\
\hline $\begin{array}{l}12 \\
\text { months }\end{array}$ & $141.78 \pm 59.94$ & $\begin{array}{l}< \\
0.05\end{array}$ & $304.62 \pm 121.47$ & $\begin{array}{l}< \\
0.05\end{array}$ & $\begin{array}{l}2323.74 \pm \\
226.45\end{array}$ & $\begin{array}{l}< \\
0.05\end{array}$ & $\begin{array}{l}1.19 \pm \\
0.27\end{array}$ & $\begin{array}{l}< \\
0.001\end{array}$ \\
\hline \multicolumn{9}{|l|}{ MT } \\
\hline Baseline & $200.96 \pm 48.48$ & & $410.59 \pm 88.53$ & & $\begin{array}{l}1350.22 \pm \\
113.84\end{array}$ & & $\begin{array}{l}1.62 \pm \\
0.21\end{array}$ & \\
\hline $\begin{array}{l}6 \\
\text { months }\end{array}$ & $135.99 \pm 26.49$ & $<001$ & $290.12 \pm 51.28$ & $\begin{array}{l}<.001 \\
0.00\end{array}$ & $1477.44 \pm 94.51$ & $\dot{0.001}$ & $\begin{array}{l}1.27 \pm \\
0.19\end{array}$ & $\begin{array}{l}< \\
0.001\end{array}$ \\
\hline $\begin{array}{l}12 \\
\text { months }\end{array}$ & $95.11 \pm 19.93$ & $\begin{array}{l}< \\
0.001\end{array}$ & $208.12 \pm 42.70$ & $<.001$ & $1596.10 \pm 96.12$ & $<.001$ & $\begin{array}{l}0.99 \pm \\
0.14\end{array}$ & $<.001$ \\
\hline \multicolumn{9}{|l|}{ LT } \\
\hline Baseline & $154.40 \pm 48.17$ & & $333.83 \pm 98.97$ & & $1384.14 \pm 92.13$ & & $\begin{array}{l}1.47 \pm \\
0.27\end{array}$ & \\
\hline
\end{tabular}




\begin{tabular}{|c|c|c|c|c|c|c|c|c|}
\hline & $\begin{array}{l}\text { Volume of } \\
\text { defective cartilage } \\
\left(\mathrm{mm}^{3}\right)\end{array}$ & $\begin{array}{l}P \\
\text { value }\end{array}$ & $\begin{array}{l}\text { Size of defective } \\
\text { cartilage }\left(\mathrm{mm}^{2}\right)\end{array}$ & $\begin{array}{l}P \\
\text { value }\end{array}$ & $\begin{array}{l}\text { Volume of } \\
\text { healthy cartilage } \\
\left(\mathrm{mm}^{3}\right)\end{array}$ & $\begin{array}{l}\mathrm{P} \\
\text { value }\end{array}$ & $\begin{array}{l}\text { Thickness } \\
\text { of } \\
\text { defective } \\
\text { cartilage } \\
(\mathrm{mm})\end{array}$ & $\begin{array}{l}P \\
\text { value }\end{array}$ \\
\hline $\begin{array}{l}6 \\
\text { months }\end{array}$ & $131.21 \pm 44.61$ & 0.087 & $283.62 \pm 89.28$ & 0.070 & $1438.02 \pm 94.16$ & 0.058 & $\begin{array}{l}1.31 \pm \\
0.24\end{array}$ & 0.030 \\
\hline $\begin{array}{l}12 \\
\text { months }\end{array}$ & $110.57 \pm 39.86$ & $\begin{array}{l}<.05 \\
0.05\end{array}$ & $238.78 \pm 81.67$ & $\begin{array}{l}< \\
0.001\end{array}$ & $1473.00 \pm 97.45$ & $\begin{array}{l}<.05 \\
0.05\end{array}$ & $\begin{array}{l}1.16 \pm \\
0.23\end{array}$ & $\begin{array}{l}< \\
0.001\end{array}$ \\
\hline \multicolumn{9}{|l|}{$P$} \\
\hline Baseline & $140.84 \pm 56.97$ & & $320.57 \pm 112.90$ & & $\begin{array}{l}1686.92 \pm \\
117.79\end{array}$ & & $\begin{array}{l}1.41 \pm \\
0.20\end{array}$ & \\
\hline $\begin{array}{l}6 \\
\text { months }\end{array}$ & $117.97 \pm 49.49$ & 0.126 & $250.71 \pm 100.31$ & 0.083 & $\begin{array}{l}1771.54 \pm \\
112.93\end{array}$ & 0.016 & $\begin{array}{l}1.23 \pm \\
0.19\end{array}$ & $\dot{0.05}$ \\
\hline $\begin{array}{l}12 \\
\text { months }\end{array}$ & $98.75 \pm 42.84$ & $\begin{array}{l}< \\
0.05\end{array}$ & $209.57 \pm 84.85$ & $\begin{array}{l}< \\
0.05\end{array}$ & $\begin{array}{l}1847.87 \pm \\
117.22\end{array}$ & $\begin{array}{l}< \\
0.001\end{array}$ & $\begin{array}{l}1.09 \pm \\
0.19\end{array}$ & $\begin{array}{l}< \\
0.001\end{array}$ \\
\hline
\end{tabular}

Similar to the patients with OA K-L grade 2 , the thickness of cartilage defect reduced in MF, LF, T, MT, LT and P, indicating a significant difference in grade OA 3. The volume of cartilage defect decreased from $278.10 \pm 110.58 \mathrm{~mm}^{3}$ to $130.30 \pm 49.56$ $\mathrm{mm}^{3}$ in MF (53\% decrease; $\left.\mathrm{p}<0.001\right)$; from $229.23 \pm 94.05 \mathrm{~mm}^{3}$ to $162.17 \pm 70.92 \mathrm{~mm}^{3}$ in LF ( $29 \%$ decrease; $\left.p<0.001\right)$; from $196.75 \pm 77.85 \mathrm{~mm}^{3}$ to $141.78 \pm 59.94 \mathrm{~mm}^{3}$ in $\mathrm{T}$ ( $28 \%$ decrease; $\left.p<0.05\right)$; from $200.96 \pm 48.48 \mathrm{~mm}^{3}$ to $95.11 \pm 19.93 \mathrm{~mm}^{3}$ in MT (53\% decrease; $p<0.001)$; from $154.40 \pm 48.17 \mathrm{~mm}^{3}$ to $110.57 \pm 39.8 \mathrm{~mm}^{3}$ in LT (28\% decrease; $\left.p<0.05\right)$; and from $140.84 \pm 56.97 \mathrm{~mm}^{3}$ to $98.75 \pm 42.84 \mathrm{~mm}^{3}$ in $\mathrm{P}(30 \%$ decrease; $p<0.05)$. Moreover, the surface of cartilage defect and volume of healthy cartilage significantly increased in all regions. However, the surface of cartilage defect decreased from $525.43 \pm 167.38 \mathrm{~mm}^{2}$ to $286.18 \pm 108.47 \mathrm{~mm}^{2}$ and from $410.59 \pm 88.53 \mathrm{~mm}^{2}$ to $208.12 \pm 42.70 \mathrm{~mm}^{2}$ in MF (46\% decrease; $p$ $<0.001)$ and MT (49\% decrease; $p<0.001)$, respectively. We also noted that the volume of healthy cartilage increased from $2382.20 \pm 314.39 \mathrm{~mm}^{3}$ to $2712.22 \pm 343.55 \mathrm{~mm}^{3}$ and from $1350.22 \pm 113.84 \mathrm{~mm}^{3}$ to $1596.10 \pm 96.12 \mathrm{~mm}^{3}$ in MF $(14 \%$ increase; $p<0.05)$ and MT (18\% increase; $p<0.05)$, respectively. In general, we believe that the effect of cartilage repair on medial cartilage was better than lateral cartilage.

\section{Discussion}

Nonoperative therapy is a frequently prescribed option for knee osteoarthritis treatment. Unfortunately, conservative treatment has been found to only temporarily relieve clinical symptoms, while their long-term efficacy is not satisfactory, eventually requiring an alternative intervention, TKA. Previous studies have highlighted that TKA may be associated with lifethreatening complications such as infection, thromboembolism, myocardial infarction, and even death. In addition, the life span of the prosthesis is between 10 and 15 years [29]. Therefore, it will be of great significance to find an effective treatment particularly for reversing the progression of this disease. The results of SVF increased the confidence of orthopedic surgeons in using nonoperative therapy to reverse the progression of OA. Interestingly, numerous studies have recently confirmed that intra-articular injection of autologous adipose-derived SVF for the treatment of OA pain is safe and feasible [30-33]. However, most clinical studies on SVF had small sample sizes, so estimates from individual studies may be imprecise, and their radiological evaluation only remains at the 2D level. So far, it remains enigmatic whether SVF can promote the growth of cartilage.

Furthermore, multiple recent studies have reported inconsistent findings of the effect of SVF on cartilage regeneration. For instance, Hong et al found that the knee joint exhibited significant defect filling and cartilage repair after receiving SVF. Similarly, WORMS and MOCART scores verified this conclusion [34]. Another study by Bansal et al followed patients who 
were injected with SVF for one year. Based on the analysis of MRI, they observed that the thickness of cartilage increased by at least $0.2 \mathrm{~mm}$ in 6 patients, indicating that there were clear signs of cartilage regeneration [16]. In 2017, Nguyen proposed that the cartilage regeneration of knee joint after AM combined with SVF/PRP injection was probably due to the combination of SVF and PRP, where SVF is the primary factor of this healing reaction [21]. Elsewhere, several studies confirmed that PRP significantly reduced short-term pain without cartilage regeneration [35-37]. In a double-blinded prospective randomized controlled clinical trial, Jaime et al reported no significant difference in cartilage thickness between the experimental group injected with SVF and the control group injected with placebo. However, in this study, participants were followed for only six months [38]. Currently, no consistent conclusion has been reached concerning whether there was obvious cartilage regeneration after intra-articular injection of SVF, hence requiring further investigation.

The emergence of 3D-FS-SPGR sequence and finite element analysis provided a new method to evaluate cartilage quantitatively. Richard et al proposed that routine MRI with a 3D sequence can improve the diagnostic performance for detecting cartilage lesions in the knee [39]. Cha believed that the 3D-SPGR sequence can provide better diagnostic performance for the evaluation of knee articular cartilage lesions by detecting partial-thickness cartilage lesions in patients with OA [40]. In 2014, Mootanah et al combined 3D-SPGR sequence with finite element analysis. Using 3D-SPGR and Mimics software, they established the model of the knee and soft tissue for accurate prediction of normal intra-articular pressure and force under different loads, which can be further developed for subject-specific surgical planning [27]. Likewise, Kaiser also developed the model of the knee joint to explore the relationship between joint mechanics and the development of OA by accurately assessing knee kinematics and cartilage contact [41]. In another report, Li studied the effect of medial meniscus degenerative tear on the progression of OA by finite element simulation. Through the establishment of meniscus and cartilage models and the analysis of biomechanics, they proposed that it may lead to cartilage injury and accelerate the progress of osteoarthritis with the aggravation of meniscus injury. Meanwhile, they also found that meniscectomy can relieve pain for some time, resulting in more severe biomechanical changes and increase the risk of OA [42]. Taken together, these studies confirmed that the 3D-SPGR sequence can provide better diagnostic performance, and the calculated cartilage model is reliable through the 3D-SPGR sequence. However, to our knowledge, no study has applied this new idea to evaluate cartilage repair.

Herein, we enrolled 47 patients with knee OA, K-L grade 2 and 3 in this study. Each patient underwent the examination of the 3D-FS-SPGR sequence before treatment, 6 and 12 months. We employed the 3D-FS-SPGR sequence to develop a 3D cartilage model, thereby dividing the cartilage at a 3D level. Compared with the conventional MRI sequence, the slice thickness of the 3D-FS-SPGR sequence was $1 \mathrm{~mm}$, which can reduce the volume effect on imaging, and consequently, the resulting data is more accurate. Following this, we recorded the changes of cartilage parameters in each region. In another study, Chris used the specific parameters of the cartilage model to assess the efficacy of MSCs in the treatment of OA but did not use a special MRI sequence, thus the appearance of the cartilage model was poor [33]. Remarkably, we noted that the cartilage of all regions was improved to some extent in our study, especially the MF and MT. In grade $2 \mathrm{OA}$, the thickness, volume, and size of cartilage defect in MF decreased from $1.53 \pm 0.23 \mathrm{~mm}$ to $0.92 \pm 0.18 \mathrm{~mm}$ (40\% decrease; $p<0.001$ ); from $173.82 \pm 63.41$ $\mathrm{mm}^{3}$ to $84.00 \pm 32.30 \mathrm{~mm}^{3}$ ( $52 \%$ decrease; $\mathrm{p}<0.001$ ); and from $353.86 \pm 122.99 \mathrm{~mm}^{2}$ to $182.22 \pm 67.00 \mathrm{~mm}^{2}$ (49\% decrease; $p<0.001)$. These parameters decreased to $0.96 \pm 0.22 \mathrm{~mm}$ (33\% decrease; $p<0.001), 64.18 \pm 21.40 \mathrm{~mm}^{3}(54 \%$ decrease; $p<$ 0.001 ), and $146.15 \pm 45.47 \mathrm{~mm}^{2}$ (48\% decrease; $\left.p<0.001\right)$ in MT. Similarly, the thickness, volume, and size of cartilage defects in MF and MT were greatly improved in grade $3 \mathrm{OA}$. We identified that the efficacy of patients with medial cartilage injury was better compared with that of patients with other region injuries, whether pain improvement, functional recovery, or cartilage repair. This finding agreed with our clinical and imaging assessments. We also uncovered that WOMAC, VAS, and WORMS scores showed better improvement in patients with medial cartilage injury.

In a multicentre analysis involving 2372 patients, postoperative pain was the major complication [43]. In this work, we observed no severe adverse events, except pain and swelling of the knee joint after the operation. Specifically, for patients with grade 2 and 3 OA, the mean WOMAC pain, stiffness, and physical function scores all revealed a significant improvement from baseline to 12 months. Similarly, the values of VAS and ROM of the joint also indicated a marked improvement in the

Page $14 / 24$ 
same category of patients. Noticeably, patients exhibited the greatest improvement at the third month compared to the baseline. Furthermore, patients revealed no significant improvement during the last follow-up compared with the third month, showing no aggravation of the symptoms. The MRI follow-up showed substantial improvements in the WORMS and MOCART scores in this study. Patients with grade 2 and 3 OA, cartilage, bone marrow abnormalities, and bone cysts exhibited a considerable improvement at 12 months, which was consistent with the results of the model parameters in our follow-up. The results of MRI also illustrated that SVF exerted a good effect on cartilage repair. Nevertheless, it exhibited no significant therapeutic effect on the osteophyte and soft tissue (menisci and ligaments) in the knee joint.

Besides, we further observed that the cartilage of patients with knee OA K-L grade 2 and 3 had different responses to SVF injection. In 2017, Nguyen found that both grade 2 and 3 OA benefited from SVF treatment, but the group of grade 2 showed a more significant increase in WOMAC and Lysholm scores [21]. This conclusion was confirmed in a more recent by Denis. They proposed that the higher the grade of the osteoarthritis, the lower the KOOS score improvement. Additionally, patients with low-grade osteoarthritis were more likely to have a higher probability of benefitting from treatment with the SVF [44]. In 2019, Tran et al came to the opposite conclusion, where they deduced that patients with grade 3 OA had better improvement after intra-articular injection of SVF than patients with grade 2 OA [22]. Here, we found that the WOMAC score, ROM, and rehabilitation speed of patients with grade 2 OA were significantly better than those of patients with grade 3 OA. On the other hand, based on the degree of cartilage repair, the increase of grade 3 OA was higher compared to that of grade 2 OA. In this present investigation, SVF suspension was not coupled with any other treatment, which can be used to evaluate its efficacy. In summary, these results suggest that SVF cell therapy can effectively improve clinical symptoms and promote cartilage regeneration before the excessive development of knee OA degeneration.

However, despite these promising results, this work has some limitations that are worth noting. First, our study had no suitable control group. In this respect, we plan to study the differences between SVF cells and other intra-articular biotherapies in the future. Second, the segmentation of the image was done through manual segmentation, which would increase some errors. In this regard, we will look for a more accurate method for the segmentation of cartilage in our future study. Then, the follow-up period was short (12 months), whereby clinical evaluations were performed at baseline, 1, 3, 6, and 12 months after intra-articular injection of SVF cells into the knee. We thus recommend that future studies should consider more follow-up time so as to determine the long-term effect of SVF. Third, we did not evaluate the relationship between the intra-articular injection dose of SVF cells and clinical results, hence the effect of dose on clinical efficacy is not clear. Therefore, more in-depth clinical trials are needed to explore the appropriate dose of SVF. Finally, although the MRI and parameters of the cartilage model clearly elucidated the regeneration of articular cartilage, it remains elusive whether the regenerated cartilage was either fibrocartilage or hyaline cartilage as arthroscopic biopsies were not possible in any of the subjects.

\section{Conclusion}

Collectively, this study demonstrates that intra-articular SVF injections can significantly reduce the symptoms and pain of knee osteoarthritis, improve function, and cause no adverse events. We also noted that the method of establishing a model and calculating parameters through the 3D-FS-SPGR sequence can accurately evaluate the effect of cartilage repair. During the follow-up period of one year, both qualitative and quantitative data showed statistically significant improvements. Therefore, this study suggests that intra-articular injection of SVF is a promising minimally invasive therapy for the treatment of knee OA, particularly for patients with $\mathrm{K}-\mathrm{L}$ grade 2 and 3.

\section{Abbreviations}

OA: Osteoarthritis; SVF: Stromal vascular fraction; MRI: Magnetic resonance imaging; 3D-FS-SPGR: Three-dimensional fatsuppressed spoiled gradient recalled echo; K-L: Kellgren Lawrence; TKA: Total knee arthroplasty; MSCs: Mesenchymal stem cells; ADSCs: Adipose-derived stem cells; WOMAC: Western Ontario and McMaster Universities Osteoarthritis Index; VAS: Visual Analog Scale; SAE: Serious adverse events; WORMS: Whole-Organ Magnetic Resonance Imaging Score; MOCART: 
Magnetic Resonance Observation of Cartilage Repair Tissue; MF: Medial femoral condyle; LF: Lateral femoral condyle; T: Femoral inter-condylar; MT: Medial tibial condyle; LT: Lateral tibia condyle; P: Patella.

\section{Declarations}

\section{Acknowledgements}

Not applicable.

\section{Availability of data and materials}

The datasets used or analyzed during the current study are available from the corresponding author on reasonable request.

\section{Authors' contributions}

The authors designed the studies and analyzed and interpreted the data. All authors have made substantial contributions: (1) the conception and design of the study, or acquisition of data, or analysis and interpretation of data; (2) drafting the article or revising it critically for important intellectual content; and (3) final approval of the version to be submitted. Qiong Zhang (email: zqzjsrmyy@163.com) takes responsibility for the integrity of the work as a whole.

\section{Funding}

This study was supported by grants from National Natural Science Foundation of China (81672769) and Zhejiang Medical and Health Science and Technology Project (Grant No. 2017KY204).

\section{Ethics approval and consent to participate}

All procedures performed in the studies involving human participants were in accordance with the ethical standards of Ethics Committee of the Zhejiang Provincial People's Hospital and with the 1964 Helsinki Declaration and its later amendments or comparable ethical standards. Researchers adhered to the principles of the Helsinki Declaration throughout the study and written informed consent was obtained from the patients. The study was registered at

http://www.chictr.org.cn/listbycreater.aspx (ChiCTR2100042930).

\section{Consent for publication}

Not applicable.

\section{Competing interests}

The authors declare that they have no competing interests.

\section{Author details}

${ }^{1}$ Rehabilitation \& sports medicine research institute of Zhejiang province, Zhejiang Provincial People's Hospital, People's Hospital of Hangzhou Medical College, Hangzhou 310014, China. ${ }^{2}$ Department of Orthopedic Surgery, Zhejiang Provincial People's Hospital and People's Hospital of Hangzhou Medical College, No.158 Shangtang Road, Hangzhou 310014, Zhejiang, China. ${ }^{3}$ Department of Operating Room, Zhejiang Provincial People's Hospital and People's Hospital of Hangzhou Medical College, No.158 Shangtang Road, Hangzhou 310014, Zhejiang, China. ${ }^{4}$ The First Affiliated Hospital of Bengbu Medical University, Bengbu, Anhui, 233004, China. ${ }^{5}$ The Second Affiliated Hospital and Yuying Children's Hospital of Wenzhou Medical University, Wenzhou, Zhejiang, 325000, China.

\section{References}


1. Loeser RF , Goldring SR, Scanzello CR, Goldring MB. Osteoarthritis: a disease of the joint as an organ. Arthritis Rheum. 2012;64:1697-707.

2. Sun HB. Mechanical loading, cartilage degradation and arthritis. Ann N Y Acad Sci. 2010;1211:37-50.

3. Wallace IJ, Worthington S, Felson DT, et al. Knee osteoarthritis has doubled in prevalence since the mid-20th century. Proc Natl Acad Sci U S A. 2017;114:9332-6.

4. Zhang W, Moskowitz R W, Nuki G et al. OARSI recommendations for the management of hip and knee osteoarthritis, Part II: OARSI evidence-based, Expert Consensus Guidelines. Osteoarthritis Cartilage. 2008;16:137-62.

5. Wehling P, Evans C, Wehling J, Maixner W. Effectiveness of intra-articular therapies in osteoarthritis: a literature review. Ther Adv Musculoskelet Dis. 2017;9:183-96.

6. Patricia A Zuk, Min Zhu, Peter Ashjian, et al. Human adipose tissue is a source of multipotent stem cells. Mol Biol Cell. 2002;13:4279-95.

7. Coughlin RP, Oldweiler A, Mickelson DT, et al. Adipose-derived stem cell transplant technique for degenerative joint disease. Arthrosc Tech. 2017;6:e1761-e1766.

8. Lamo-Espinosa JM, Mora G, Blanco JF, et al. Intra-articular injection of two different doses of autologous bone marrow mesenchymal stem cells versus hyaluronic acid in the treatment of knee osteoarthritis: multicenter randomized controlled clinical trial (phase I/II). J Transl Med. 2016;14:246.

9. Lindroos B, Suuronen R, Miettinen S. The potential of adipose stem cells in regenerative medicine. Stem Cell Rev Reports. 2011;7:269-91.

10. Philippe Bourin, Bruce A Bunnell, Louis Casteilla, et al. Stromal cells from the adipose tissue-derived stromal vascular fraction and culture expanded adipose tissue-derived stromal/stem cells: a joint statement of the International Federation for Adipose Therapeutics and Science (IFATS) and the International Society for Cellular Therapy (ISCT). Cytotherapy. 2013;15:641-8.

11. Han J, Koh YJ, Moon HR, et al. Adipose tissue is an extramedullary reservoir for functional hematopoietic stem and progenitor cells. Blood. 2010;115:957-64.

12. McIntosh K, Zvonic S, Garrett S, et al. The immunogenicity of human adipose-derived cells: temporal changes in vitro. Stem Cells. 2006;24:1246-53.

13. Feng Z, Ting J, Alfonso Z, Strem BM, et al. Fresh and cryopreserved, uncultured adipose tissue-derived stem and regenerative cells ameliorate ischemia-reperfusion- induced acute kidney injury. Nephrol Dial Transplant. 2010;25:387484.

14. Jeffrey M Gimble, Adam J Katz, Bruce A Bunnell. Adipose-derived stem cells for regenerative medicine. Circ Res. 2007;100:1249-60.

15. Rehman J, Traktuev D, Li J, et al. The secretion of angiogenic and anti-apoptotic factors by human adipose stromal cells. Circulation. 2004;109:1291-8.

16. Bansal H, Comella K, Leon J, et al. Intra-articular injection in the knee of adipose-derived stromal cells (stromal vascular fraction) and platelet rich plasma for osteoarthritis. J Transl Med. 2017;15:141.

17. Koh YG, Choi YJ, Kwon SK, et al. Clinical results and second-look arthroscopic findings after treatment with adiposederived stem cells for knee osteoarthritis. Knee Surg Sports Traumatol Arthrosc. 2015;23:1308-16.

18. Koh YG, Jo SB, Kwon OR, et al. Mesenchymal stem cell injections improve symptoms of knee osteoarthritis. Arthroscopy. 2013;29:748-55.

19. Yokota N, Yamakawa M, Shirata T, et al. Clinical results following intra-articular injection of adipose-derived stromal vascular fraction cells in patients with osteoarthritis of the knee. Regen Ther. 2017;6:108-12.

20. Michalek, J, Moster, R, Lukac, L, et al. Stromal Vascular Fraction Cells of Adipose and Connective Tissue in People with Osteoarthritis: A Case Control Prospective Multi-Centric Non-Randomized Study. Glob Surg. 2017;3:1-9.

Page $17 / 24$ 
21. Nguyen PD, Tran TD, Nguyen HT, et al. Comparative Clinical Observation of Arthroscopic Microfracture in the Presence and Absence of a Stromal Vascular Fraction Injection for Osteoarthritis. Stem Cells Transl Med. 2017;6(1):187-95.

22. Tung Dang Xuan Tran, Chi-Ming Wu, Navneet Kumar Dubey, et al. Time-and Kellgren-Lawrence Grade-Dependent Changes in Intra-Articularly Transplanted Stromal Vascular Fraction in Osteoarthritic Patients. Cells. 2019;8(4):308.

23. Peter B Fodor, Stephen G Paulseth.Adipose Derived Stromal Cell (ADSC) Injections for Pain Management of Osteoarthritis in the Human Knee Joint. Aesthetic Surg J. 2016;36:229-36.

24. Kellgren JH, Lawrence JS. Radiological assessment of osteoarthrosis. Ann Rheum Dis. 1957;16:494-502.

25. Peterfy CG, Guermazi A, Zaim S, et al. Genant HK (2004) Whole-organ magnetic resonance imaging score (WORMS) of the knee in osteoarthritis.Osteoarthr Cartilage. 2004;12:177-90.

26. Marlovits S, Striessnig G, Resinger CT, et al. Definition of pertinent parameters for the evaluation of articular cartilage repair tissue with high-resolution magnetic resonance imaging. Eur J Radiol. 2004;52:310-9.

27. R Mootanah , C W Imhauser, F Reisse, et al. Development and Validation of a Computational Model of the Knee Joint for the Evaluation of Surgical Treatments for Osteoarthritis. Comput Methods Biomech Biomed Engin. 2014;17:1502-1517.

28. Xinxin Zhao, Jingjing Ruan, Hui Tang, et al. Multi-compositional MRI Evaluation of Repair Cartilage in Knee Osteoarthritis With Treatment of Allogeneic Human Adipose-Derived Mesenchymal Progenitor Cells. Stem Cell Res Ther. 2019;10:308.

29. Schrama JC, Espehaug B, Hallan G, et al. Risk of revision for infection in primary total hip and knee arthroplasty in patients with rheumatoid arthritis compared with osteoarthritis: a prospective, population-based study on 108,786 hip and knees joint arthroplasties from the Norwegian Arthoplasty Register. Arthritis Care Res. 2010;62:473-9.

30. Orozco L, Munar A, Soler R, et al. Treatment of knee osteoarthritis with autologous mesenchymal stem cells: a pilot study. Transplantation. 2013;95:1535-41.

31. Davatchi F, Abdollahi BS, Mohyeddin M, et, al. Mesenchymal stem cell therapy for knee osteoarthritis. Preliminary report of four patients. Int J Rheum Dis. 2011;14:211-5.

32. Kim YS, Choi YJ, Lee SW, et al. Assessment of clinical and MRI outcomes after mesenchymal stem cell implantation in patients with knee osteoarthritis: a prospective study. Osteoarthritis Cartilage. 2016;242:237-45.

33. Jo CH, Lee YG, Shin WH, et al. Intra-articular injection of mesenchymal stem cells for the treatment of osteoarthritis of the knee: a proof-of-concept clinical trial. Stem Cells. 2014;32:1254-66.

34. Hong Z, Chen J, Zhang S, et al. Intra-articular injection of autologous adipose-derived stromal vascular fractions for knee osteoarthritis: a double-blind randomized self-controlled trial.Int Orthop. 2019;43:1123-34.

35. Ayhan E, Kesmezacar H, Akgun I. Intraarticular injections (corticosteroid, hyaluronic acid, platelet rich plasma) for the knee osteoarthritis. World J Orthop. 2014;5:351-61.

36. Alberto Gobbi, Georgios Karnatzikos, Vivek Mahajan, et, al. Platelet-rich plasma treatment in symptomatic patients with knee osteoarthritis: Preliminary results in a group of active patients. Sports Health. 2012;4:162-72.

37. Filardo G, Kon E, Buda R et al. Platelet-rich plasma intra-articular knee injections for the treatment of degenerative cartilage lesions and osteoarthritis. Knee Surg Sports Traumatol Arthrosc. 2011;19:528-35.

38. Garza JR, Campbell RE, Tjoumakaris FP, et al. Clinical Efficacy of Intra-articular Mesenchymal Stromal Cells for the Treatment of Knee Osteoarthritis A Double-Blinded Prospective Randomized Controlled Clinical Trial.Am J Sports Med. 2020;483:588-98.

39. Richard Kijowski, Donna G Blankenbaker, Michael Woods, et al. Clinical usefulness of adding 3D cartilage imaging sequences to a routine knee MR protocol. AJR Am J Roentgenol. 2011;196:159-67.

40. Jang Gyu Cha, Jae Ho Yoo, Sun Jung Rhee, et al. MR imaging of articular cartilage at 1.5T and 3.0T: comparison of IDEAL 2D FSE and 3D SPGR with fat-saturated 2D FSE and 3D SPGR in a porcine model. Acta Radiol. 2014;55:462-9.

41. Jarred Kaiser, Arezu Monawer, Rajeev Chaudhary, et al. Accuracy of model-based tracking of knee kinematics and cartilage contact measured by dynamic volumetric MRI. Med Eng Phys. 2016;38:1131-5. 
42. Lan Li, Longfei Yang, Kaijia Zhang, et al. Three-dimensional finite-element analysis of aggravating medial meniscus tears on knee osteoarthritis. J Orthop Translat. 2019;20:47-55.

43. Centeno CJ, Al-Sayegh H, Freeman MD, et al. A multi-center analysis of adverse events among two thousand, three hundred and seventy two adult patients undergoing adult autologous stem cell therapy for orthopedic conditions. Int Orthod. 2016;40(8):1755-65.

44. Denis Simunec, Honey Salari, Juliane Meyer. Treatment of Grade 3 and 4 Osteoarthritis with Intraoperatively Separated Adipose Tissue-Derived Stromal Vascular Fraction: A Comparative Case Series. 2020;9:2096.

\section{Figures}
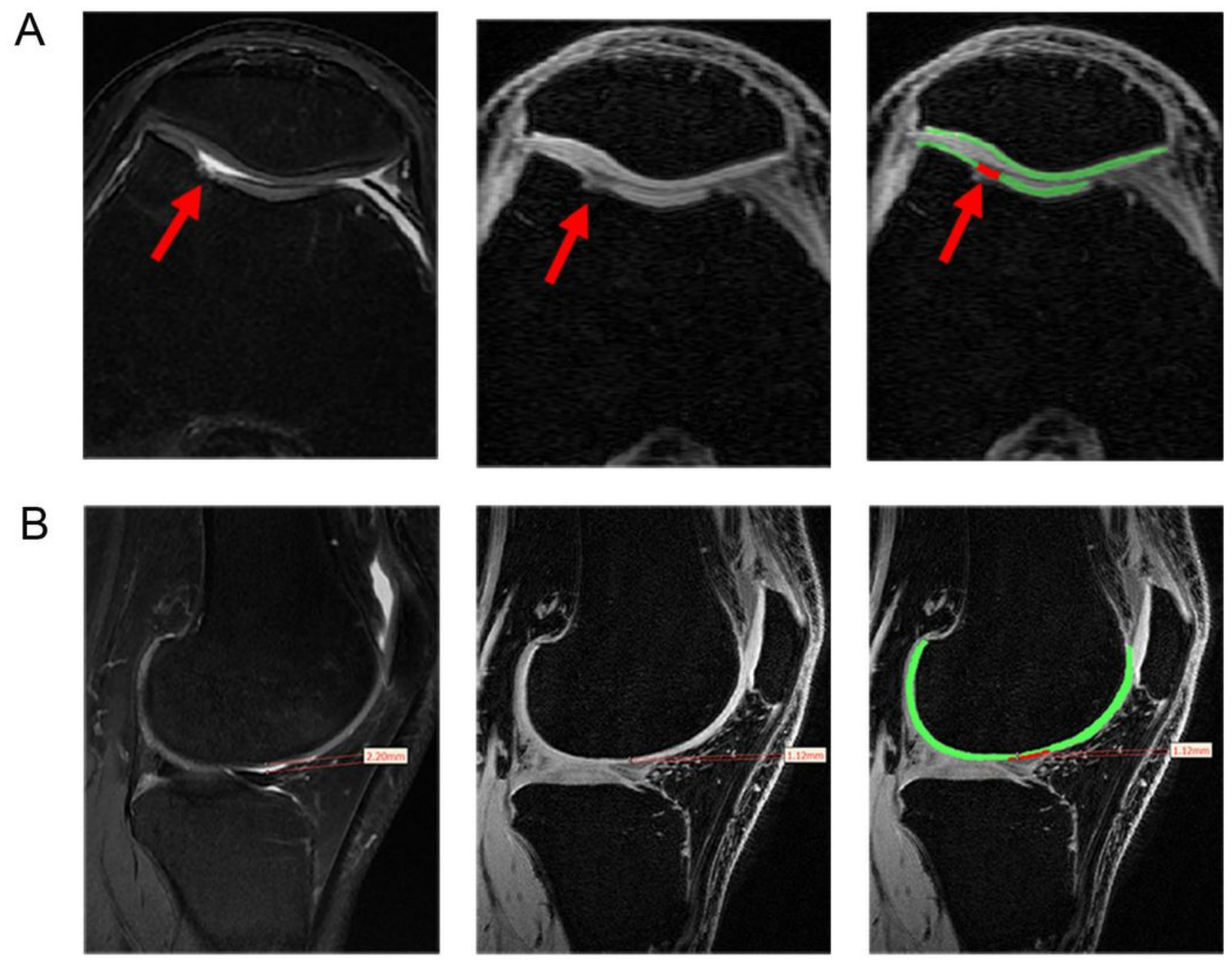

\section{Figure 1}

Shows different color masks used to distinguish healthy cartilage from cartilage defects by setting the threshold. Illustrating the injury of the whole layer of cartilage (a) and partial cartilage defects (b). 
A

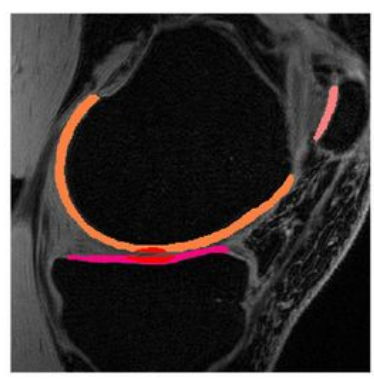

B

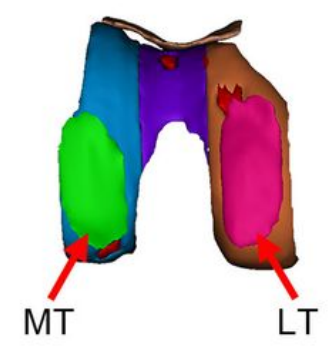

$\mathrm{C}$

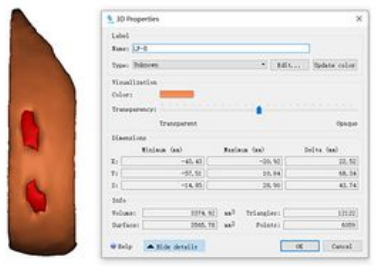

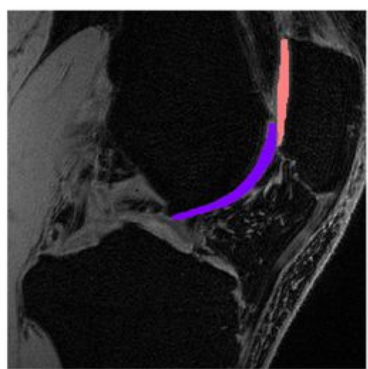
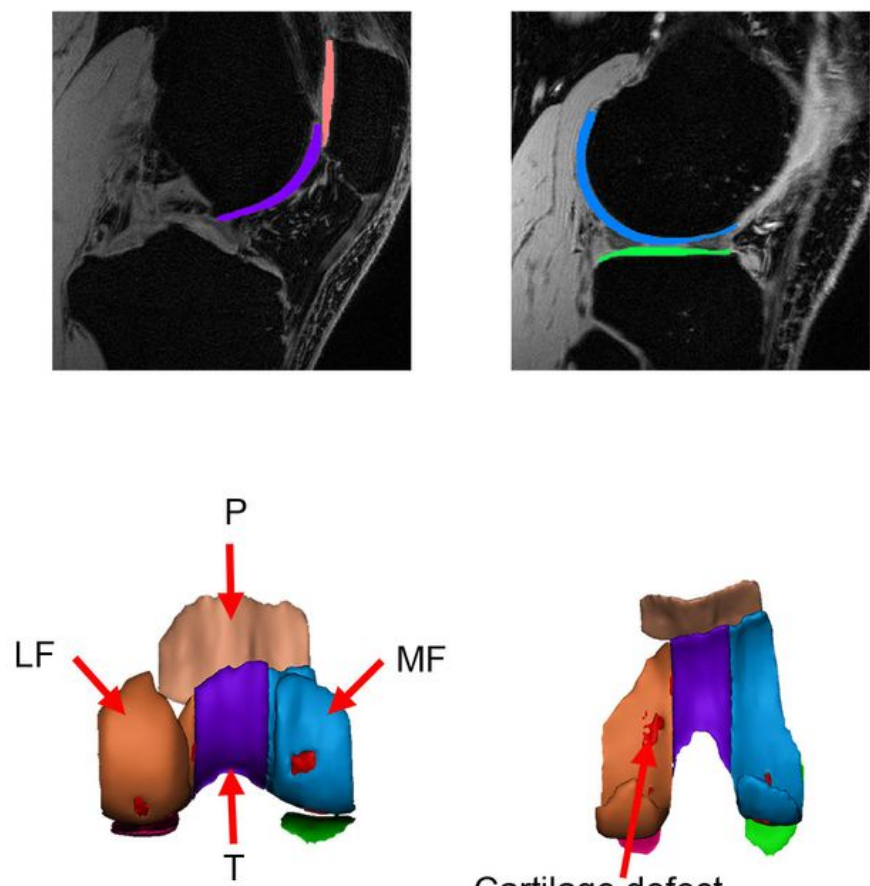

Cartilage defect

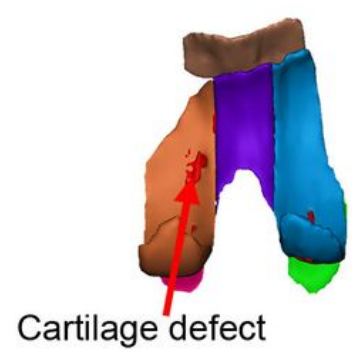

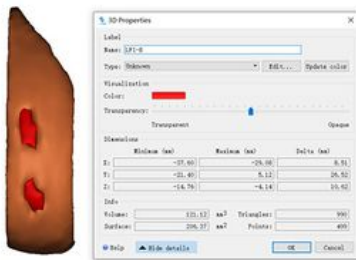
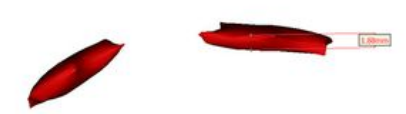

\section{Figure 2}

The cartilage of the knee joint divided into six regions with different color masks, namely, lateral femoral condyle (LF), femoral inter-condylar (T), medial femoral condyle (MF), lateral tibia condyle (LT), medial tibia condyle (MT) and patella (P) (a). After this, the knee cartilage model was established (b) and the parameters of model were measured (For example: In lateral femoral condyle, the thickness, volume and surface of cartilage defect were $1.88 \mathrm{~mm}, 121.12 \mathrm{~mm} 3 \mathrm{and} 206.37 \mathrm{~mm} 2$, respectively. The volume of healthy cartilage was $3374.92 \mathrm{~mm} 3$ (c)). 


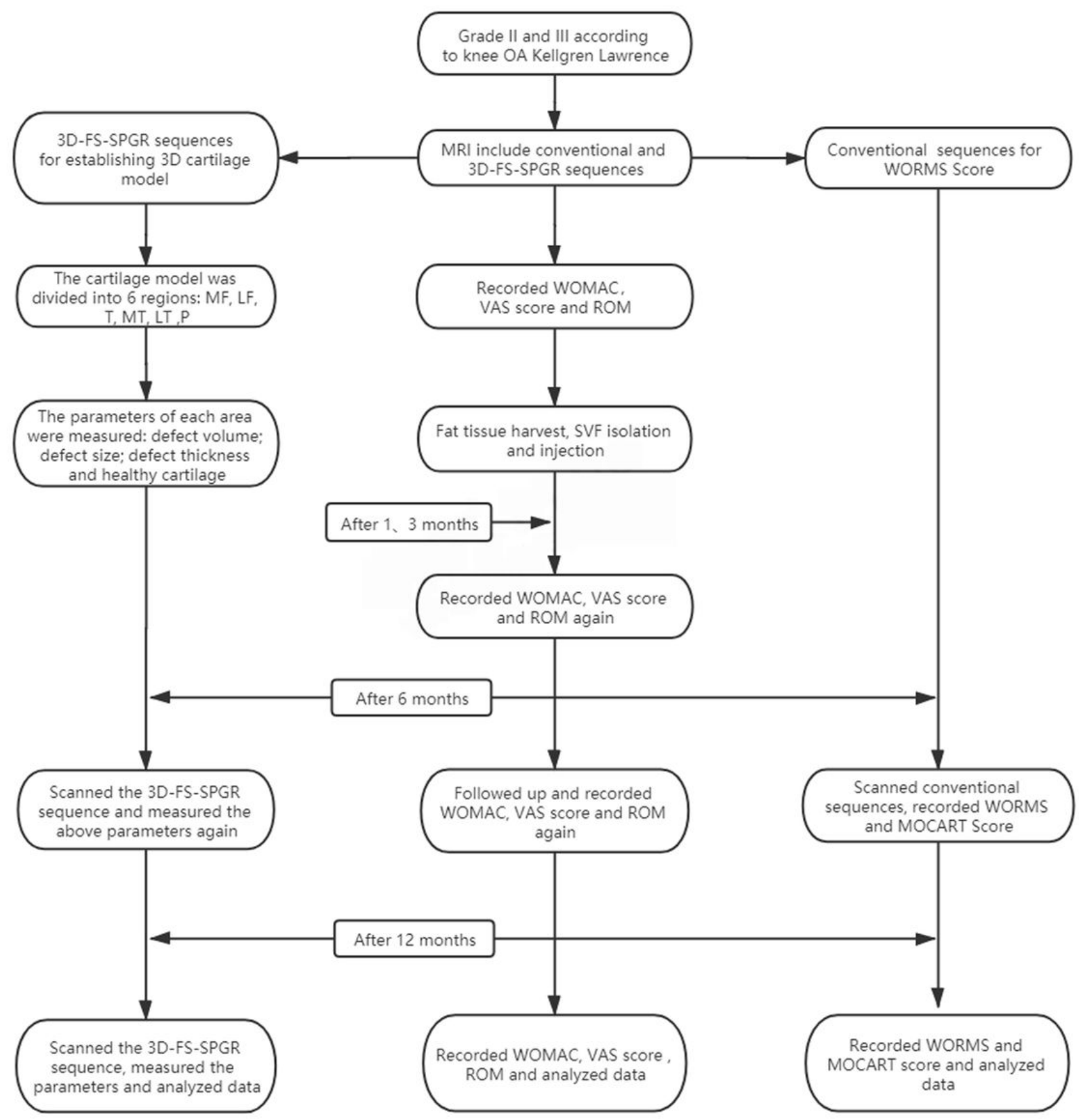

Figure 3

Flowchart of the clinical trial. 
A

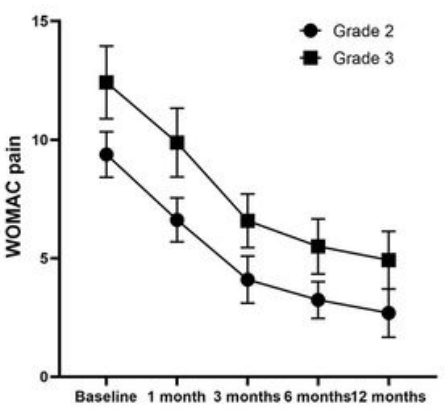

B

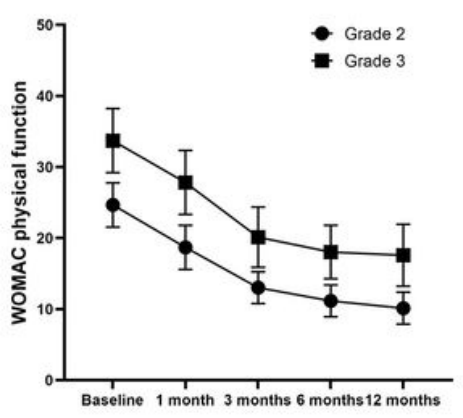

$\mathrm{C}$

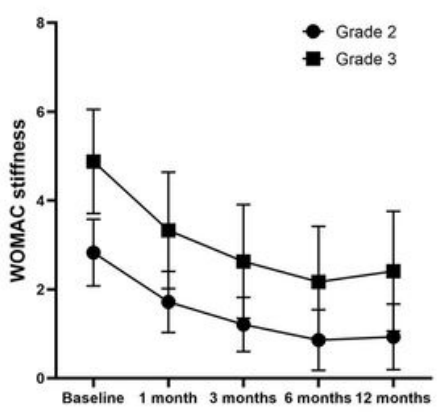

$\mathrm{D}$

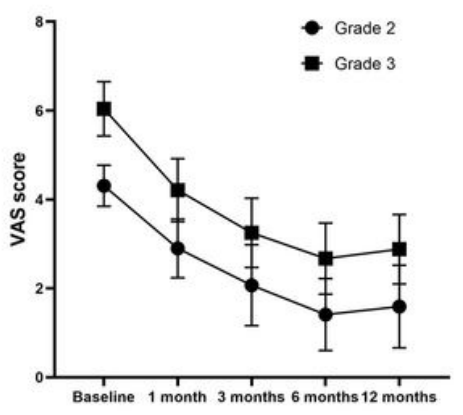

$\mathrm{E}$

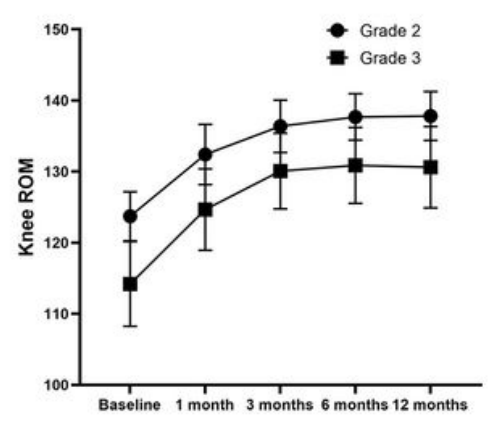

Figure 4

Changes of the VAS, ROM, WOMAC pain, stiffness, and physical function during 12-months follow-up after intra-articular injection of SVF. The WOMAC pain, stiffness, and physical function scores indicating a tendency of improvement from baseline to 12 months, whereby the greatest improvement occurred at the third month $(a, b, c)$. The VAS score exhibited a significant improvement at 12 months (d). The ROM improved significantly over time, which showed a statistically significant difference from baseline to 12 months (e). 

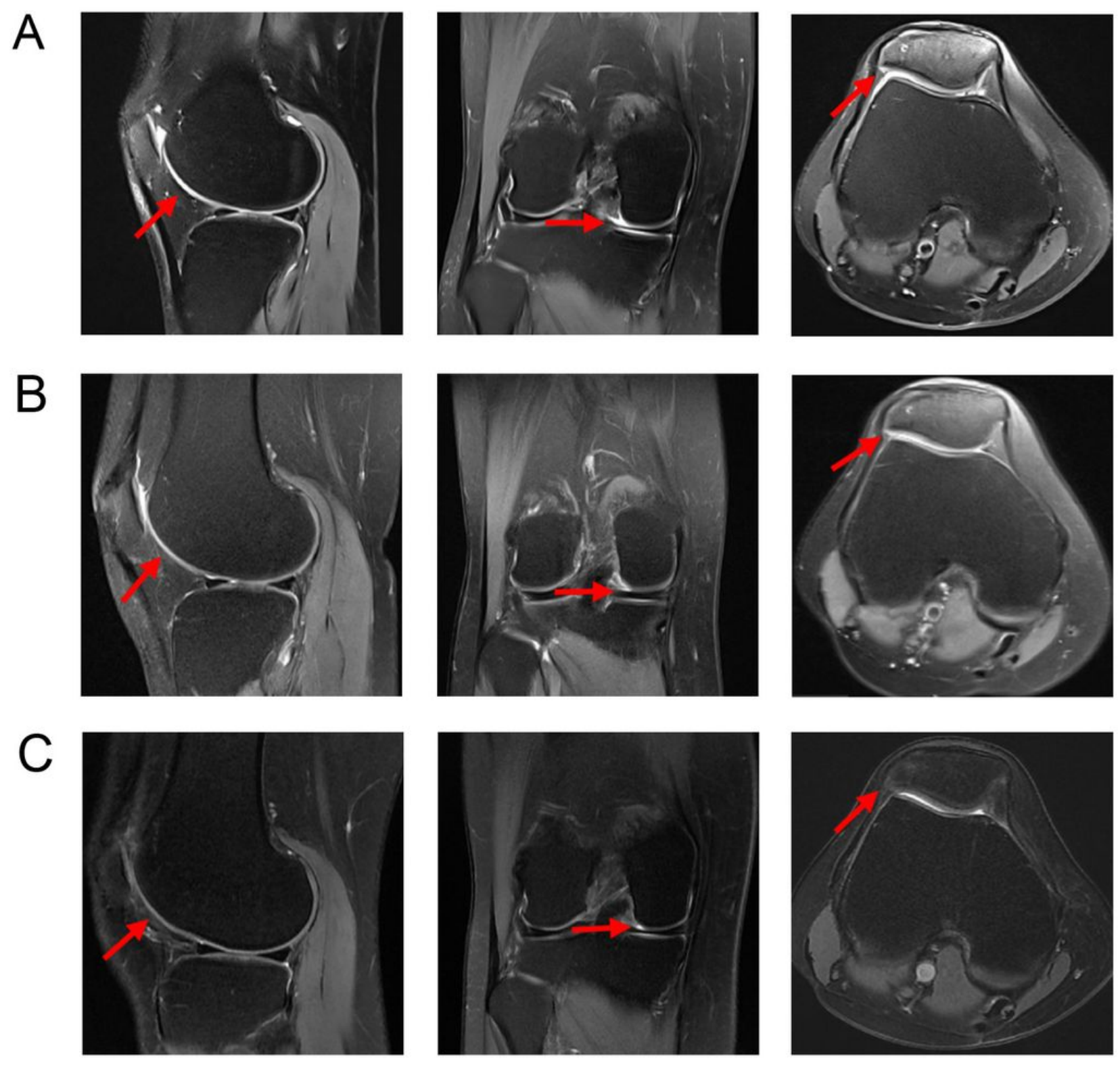

Figure 5

Shows MRI scans of three SVF-treated knees performed at baseline (a), 6 (b), and 12 months (c), respectively. It was found that the defect was completely repaired and filled, and the cartilage fused well with adjacent cartilage and subchondral bone in the coronal, transverse, and sagittal planes (red arrow). 


\section{Basline 6 months 12 months}

A
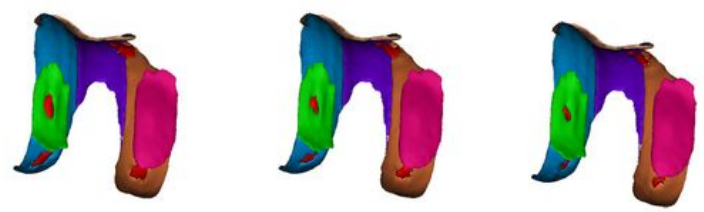

B
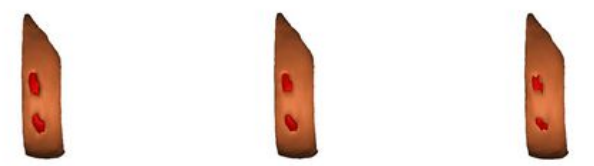

C
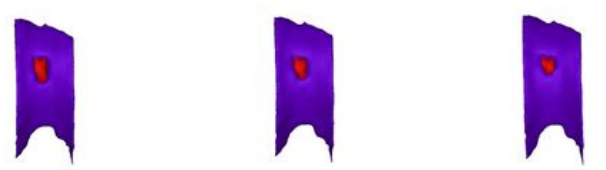

E
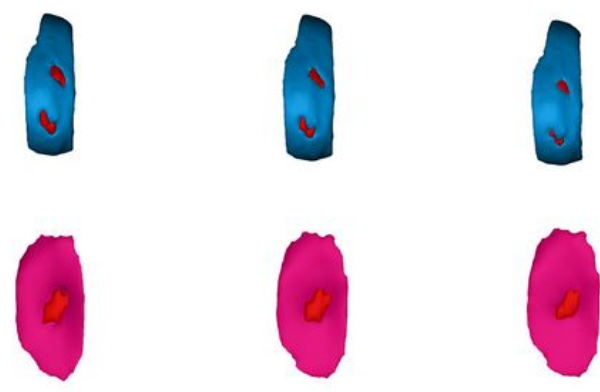

$\mathrm{F}$
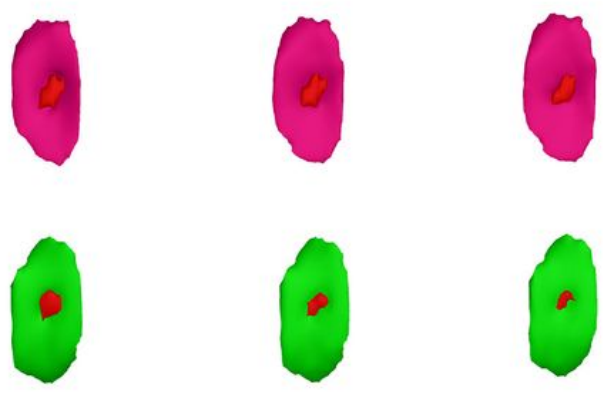

G
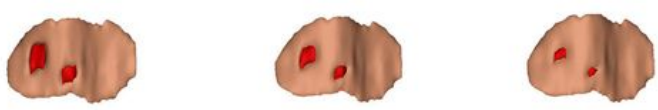

\section{Figure 6}

Cartilage model of SVF-treated knee established at baseline, 6 , and 12 months. The cartilage defect of the knee joint showed good repair (a). Change of cartilage defects in the LF at baseline, 6 months and 12 months after injection (b); Change of cartilage defects in the T at baseline, 6 months and 12 months after injection (c); Change of cartilage defects in the MF at baseline, 6 months and 12 months after injection (d); Change of cartilage defects in the LT at baseline, 6 months and 12 months after injection (e); Change of cartilage defects in the MT at baseline, 6 months and 12 months after injection (f); Change of cartilage defects in the $P$ at baseline, 6 months and 12 months after injection (g). Among them, the repair of cartilage defects in MT and MF were the best. 